\title{
Évaluation et analyse de la performance par accélérométrie lors des mouvements de musculation
}

\author{
Boris Jidovtseff $^{1}$, Guillaume Laffaye ${ }^{2}$ \\ 1. Training and assessment of physical fitness \\ Department of Sport and Rehabilitation Sciences \\ University of Liege, Allée des sports 4 \\ 400 Liège, Belgique \\ b.jidovtseff@ulg.ac.be \\ 2. UR CIAMS - Motor Control and Perception Group, Sport Sciences Department, \\ Bât. 335, Université Paris-Sud \\ 91405 Orsay, France \\ guillaume.laffaye@u-psud.fr
}

RÉSUMÉ. Depuis une décennie l'accélérométrie permet d'effectuer des mesures hors du laboratoire en biomécanique du sport. Petits, transportables, légers, les accéléromètres constitueraient une solution crédible aux lourdes plateformes de force et offriraient de nouvelles perspectives pour une meilleure compréhension de la performance sportive. Cet article présente l'accélérométrie, ses possibilités mais aussi ses limites actuelles dans le cadre de l'évaluation de la performance lors de mouvements de musculation (ex. développécouché, squat). Les accéléromètres sont utilisés lors d'exercices de renforcements musculaires pour déterminer des profils «force-vitesse-puissance », estimer la charge que l'athlète est capable de soulever en une fois (1RM), réaliser des analyses biomécaniques ou encore détecter les mouvements exécutés lors d'une séance de musculation. Ces mesures peuvent être très utiles pour le scientifique et pour l'entraîneur dans la mise en place d'un plan d'entraînement ou du suivi de l'athlète.

ABSTRACT. Since a decade, the accelerometry allows new measurements in biomechanics of sport. Small, light and transportable, these sensors allow to liberate from heavy force plates and offers new perspectives in the understanding of the performance in sport. The goal of this chapter is to present the concept of the accelerometry and to understand practical possibilities and limits in the assessment of muscular performance. The reliability of the accelerometers are highly depending on the level of technology and in the respect of manufacturer recommendations. These devices are used during exercises such as bench press or squat, allowing to analyze force-velocity-power profile, to estimate the 1-RM, to achieve biomechanical analysis or to track free weight exercises during work out. All of these applications are very precious for scientists and for coaches during training program or athletes follow-up. 
MOTS-CLÉS : accéléromètre, validité, reproductibilité, évaluation inertielle, développé couché, squat, force, puissance, profil force-vitesse, biomécanique.

KEYWORDS: accelerometer, validity, reliability, inertial assessment, bench press, squat, strength, power, force-velocity, biomechanics.

DOI:10.3199/I2M.14.3-4.197-227 @ 2014 Lavoisier

\section{Introduction}

L'accéléromètrie a envahi notre quotidien. On la retrouve dans les téléphones, les consoles de jeu, les montres, les coussins de sécurité dans les voitures, les caméras pour stabiliser l'image, etc. Un accéléromètre est un capteur qui mesure l'accélération subie par la structure où il est ancré, selon un, deux ou trois axes, qui définissent le référentiel propre au capteur. L'unité de mesure internationale de l'accélération est le m.s. ${ }^{-2}$, mais de nombreux constructeurs l'expriment préférentiellement en nombre de " $\mathrm{g}$ ", renvoyant à l'accélération gravitationnelle $\left(\mathrm{g}=9,81 \mathrm{~m} \cdot \mathrm{s}^{-2}\right)$.

Leur coût n’a cessé de baisser, le démocratisant et permettant d'avoir du matériel léger, transportable et facile d'utilisation. Son prix varie de quelques euros à plusieurs milliers d'euros pour un capteur asservi haut de gamme. On estime aujourd'hui qu'il se vend plus de 100 millions d'accéléromètres chaque année.

Dans les domaines de l'évaluation et de l'analyse d'une performance son intérêt ne cesse de grandir. Les accéléromètres sont légers, transportables, peu encombrants ce qui permet de faire de l'analyse de mouvement partout, ou presque, en laboratoire comme sur les lieux de l'entraînement. Cette particularité permet d'étudier le mouvement humain aussi bien dans des conditions très standardisées de laboratoire que dans des mouvements fonctionnels réalisés sur le lieu même d'entraînement. Toutefois certaines précautions indispensables sont à prendre car dans la plupart des cas, l'accéléromètre ne nous informe que de ce qui se passe là où le capteur est fixé. L'information offerte reste donc incomplète et il est important d'en tenir compte dans l'interprétation des résultats.

\section{L’accélérométrie}

Il existe un très grand nombre d'accéléromètres commercialisés, toutefois les modèles les plus adaptés à l'étude du mouvement humain sont les accéléromètres à gauge de contraintes piezorésistifs, piezoélectriques et capacitifs (Chen et al., 2005 ; Kavanagh et al., 2008). Le mécanisme de base permettant la mesure de l'accélération repose sur le principe du modèle masse-ressort qui répond à la loi de Hooke $(\mathrm{F}=k x)$ et à la deuxième loi de Newton $(\mathrm{F}=m a)$. Lorsqu'un système masseressort est soumis à une compression ou une force d'étirement liée au mouvement, le ressort s'allonge ou se raccourcit proportionnellement au niveau de force. Étant 
donné que la masse $(m)$ et la constante du ressort $(k)$ présentent des caractéristiques contrôlables et stables, l'accélération responsable du déplacement de la masse peut être déterminée à partir de son déplacement $(\Delta x)(1)$ :

$$
\mathrm{F}=k \Delta x=m a, \quad \text { donc } \quad a=\frac{k \Delta x}{m} a
$$

(a)

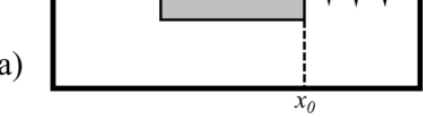

Au repos

Axe de mesure de l'accéléromètre

(b)

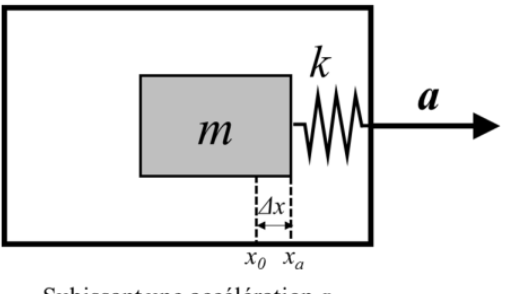

Subissant une accélération

Figure 1. Représentation schématique d'un accéléromètre selon le système masse-ressort en position de repos (a) et subissant une accélération a (b)

En réalité, l'accélération est quantifiée à partir de différentes techniques dépendantes de la catégorie de l'accéléromètre. Par exemple, dans un accéléromètre capacitif, on mesure le déplacement de la masse inertielle en mesurant la variation de capacité entre deux électrodes. Quel que soit son mode de fonctionnement, un accéléromètre fournit toujours un signal sortant sous forme de courant dont l'intensité est proportionnelle à l'accélération. La relation entre le signal électrique et la valeur de référence de l'accélération est cependant très variable et doit être déterminée par une procédure de calibration spécifique (Kavanagh et al., 2008).

De manière synthétique deux calibrations peuvent être réalisées : une calibration statique et une calibration dynamique. La calibration statique qui consiste à comparer l'accélération mesurée dans différentes positions statiques à une accélération connue et constante. La gravité est très souvent utilisée comme référentiel. Un accéléromètre positionné verticalement doit ainsi indiquer une accélération de -1g. Positionné à l'envers, il indiquera une accélération de +1g. La régression linéaire entre l'accélération mesurée et l'accélération connue permet de réaliser une compensation mathématique correctrice. Cette approche suppose une linéarité entre l'accélération et l'intensité du signal. La plupart des accéléromètres triaxiaux récents bénéficient d'une autocalibration performante (Frosio et al., 2009 ; Luinge et al., 2004). La calibration dynamique (ou périodique) prend plus de temps et nécessite un matériel spécifique tel un shaker. Cet instrument induit à l'accéléromètre une accélération harmonique qui est mise en relation avec l'accélération offerte par le capteur (Kavanagh et al., 2008). 
Une des limites des outils de mesure développés et commercialisés pour certaines applications spécifiques est qu'ils proposent souvent des données déjà traitées avec des algorithmes et des hypothèses que l'on doit accepter sans possibilité de vérification. D’un point de vue scientifique ce genre de traitement apparaît problématique. Dans un souci de transparence, il conviendrait de pouvoir s'affranchir de ces logiciels " commerciaux » et/ou d'accéder aux données brutes enregistrées par l'accéléromètre afin de permettre les comparaisons avec d'autres outils de mesure. Toutefois, des mises en situations expérimentales peuvent aider l'utilisateur à vérifier certaines de ces hypothèses. Nous l'y invitons fermement.

On distingue les accéléromètres asservis (AC-coupled) et non asservis (DCcoupled). Cette deuxième catégorie d'accéléromètres est généralement utilisée dans les applications sportives et biomécaniques. Ces capteurs permettent de détecter aussi bien l'accélération statique liée à la gravité que les composantes inertielles liées à un mouvement. Dans des conditions statiques, un accéléromètre uniaxial peut être utilisé pour mesurer une inclinaison (Moe-Nilssen et al., 2002 ; Veltink et al., 1996). En effet, en l'absence de mouvement et lorsque son axe de polarisation est positionné à la verticale, il mesurera une accélération de $-1 \mathrm{~g}$ (figure $2 \mathrm{~b}$ ) alors que positionné à l'horizontal il ne détectera aucune accélération (figure 2a). A partir d'un calcul trigonométrique simple il est facile de mesurer son inclinaison sur base de l'accélération obtenue en position statique (figure 2c). L'accéléromètre uniaxial ne permet cependant pas de définir l'orientation de cette inclinaison.

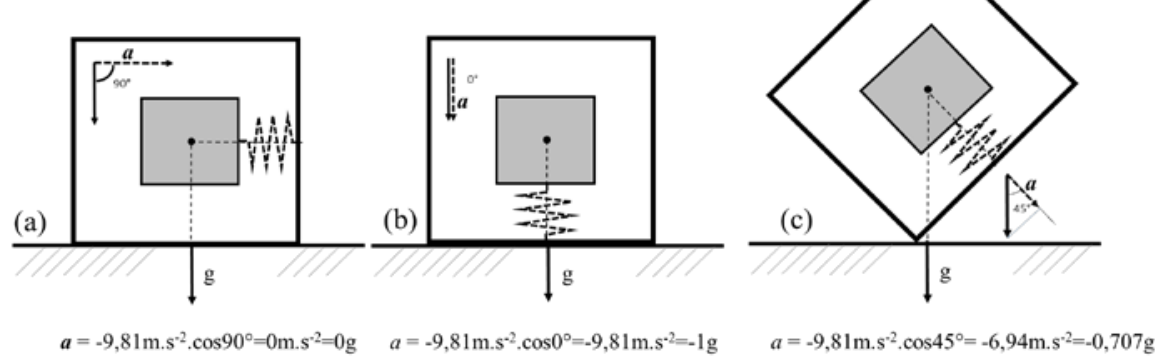

Figure 2. Mesure de l'inclinaison à partir d'un accéléromètre uniaxial

Dans des conditions dynamiques un accéléromètre uniaxial placé à l'horizontal et subissant des mouvements horizontaux offrira un signal directement proportionnel au mouvement subi (figure 3a). Si ce capteur n'est pas parfaitement positionné dans l'axe de mesure, le signal sortant sera la combinaison de l'accélération liée au mouvement et de l'accélération liée à la gravité (figure 3b). Par ailleurs, si l'accéléromètre n'est pas déplacé dans l'axe de mesure seule la composante accélératrice dans l'axe de mesure sera mesurée (figure 3c). 


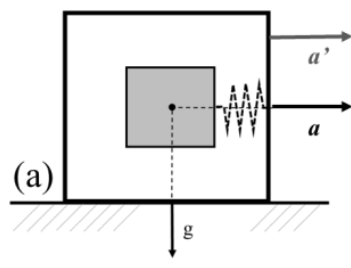

$a^{\prime}=a+\mathrm{g} \cdot \cos 90^{\circ}=a$

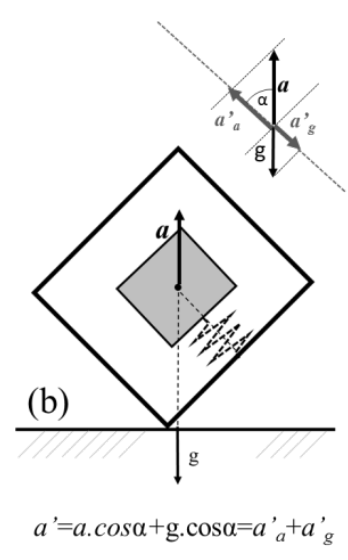

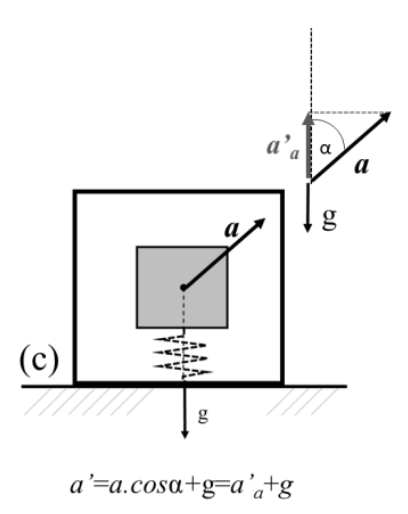

Figure 3. Mesure de l'accélération subie par un accéléromètre uniaxial placé dans l'axe du déplacement (a) par un accéléromètre incliné selon un angle a (b) et par un accéléromètre déplacé en dehors de son axe de mesure (c)

L'accéléromètre triaxial (figure 4) palie une partie de ces problèmes en permettant de mesurer l'accélération subie par l'objet dans chacun des trois axes orthogonaux. Dans des conditions statiques, la mesure de la composante gravitationnelle subie dans chaque axe permet de définir précisément l'inclinaison et l'orientation du capteur. Cette approche est d'ailleurs exploitée dans l'exploration du contrôle postural (Mayagoitia et al., 2002a ; Moe-Nilssen et al., 2002).

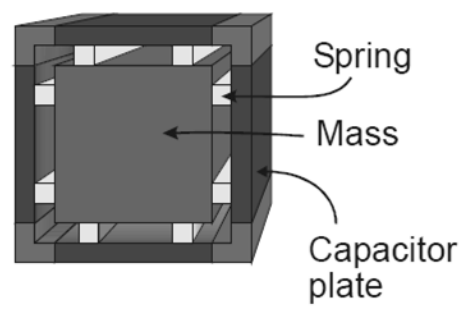

Figure 4. Représentation schématique d'un accéléromètre triaxial (Luinge, 2002)

En condition dynamique, un accéléromètre triaxial ne pourra mesurer l'accélération subie dans les trois axes avec précision que s'il ne subit pas de rotation. En effet, nous avons vu plus haut que tout changement d'orientation du capteur modifie le signal de chacune des trois composantes. Dans un mouvement dynamique avec rotation du capteur il est extrêmement compliqué de dissocier à l'intérieur de chaque signal obtenu (un par axe de l'accéléromètre) la fraction de l'accélération qui est due au mouvement, à la gravité et à la rotation (Luinge, 2002b ; Luinge et al., 2004). C'est la raison pour laquelle lorsque l'on souhaite avoir 
des informations sur les accélérations dans chaque axe d'un individu qui réalise un mouvement complexe avec rotation, il recommandé de combiner accéléromètre et gyroscope (Luinge, 2002). Cette combinaison, appelée centrale inertielle, est de plus en plus utilisée dans l'analyse biomécanique des mouvements humains car elle permet grâce au gyroscope de connaître à tout moment l'orientation exacte du capteur par rapport à un système de référence. Les angles d'Euler offerts par le gyroscope sont utilisés dans une matrice qui permet de connaître l'orientation exacte du capteur et différencier la part d'accélération, de gravité et de rotation dans chaque signal et surtout de reconstituer les accélérations du sujet dans chacun des axes de référence.

Ces centrales inertielles représentent certainement l'avenir de l'analyse de mouvement par accélérométrie. Plusieurs travaux encourageants confirment que cette technologie pourrait devenir une alternative économique très performante aux systèmes optiques d'analyse du mouvement en trois dimensions (Mayagoitia et al., 2002b). La capacité de mesure des gyroscopes et de leur échantillonnage constitue cependant encore une limite, particulièrement dans les mouvements dynamiques (Jidovtseff et al., 2010). Les caractéristiques et possibilités des différents accéléromètres sont reprises dans le tableau 1.

Pour évaluer et analyser la performance lors des mouvements de musculation, un enjeu technologique majeur est de proposer des capteurs petits, légers, peu encombrants et performants pouvant être fermement fixés à une barre de musculation ou au corps. Les systèmes intégrés et sans fil apparaissent particulièrement intéressants pour l'analyse des gestes sportifs (Intille et al., 2012). On y distingue deux grandes approches qui présentent chacune des avantages et des inconvénients. Il y a d'abord les systèmes qui enregistrent des données sur une carte mémoire intégrée (data logger). Cette approche autorise des performances techniques très intéressantes en termes de qualité de signal et d'échantillonnage. Elle permet aussi de faire des tests n'importe où : en laboratoire, salle de sport, et à l'extérieure. Par contre l'accès aux résultats est différé car il nécessite un transfert des données vers une interface informatique qui ne peut être réalisé qu'à la fin des mesures. De plus certaines erreurs de manipulation ne sont pas nécessairement visibles au moment des tests et ne seront constatées qu'au moment de la lecture des résultats. A côté de cette approche les firmes essaient de plus en plus de développer des centrales inertielles sans fil qui proposent une transmission et une lecture directe du mouvement sportif sur une interface informatique, ce qui présente de nombreux avantages. Le mouvement peut être directement analysé et l'interprétation d'un mouvement peut se faire en temps opérateur. Les erreurs sont plus facilement détectées. Par contre la transmission directe des données sans fil peut conduire à des pertes de données et par conséquent une difficulté de synchronisation avec d'autres mesures, à un échantillonnage limité, ce qui peut s'avérer problématique dans le cadre de mouvements dynamiques qui nécessitent des fréquences d'acquisition élevées. Ces systèmes sont également généralement plus onéreux. 
Tableau 1. Caractéristiques, possibilités de mesures et recommandations d'utilisation des différents accéléromètres dans l'évaluation d'un soulever de barre

\begin{tabular}{|c|c|c|c|}
\hline & Accéléromètre uniaxial & Accéléromètre triaxial & Centrale inertielle \\
\hline 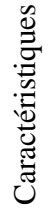 & $\begin{array}{c}\text { Accélération dans l'axe } \\
\text { Z }\end{array}$ & $\begin{array}{l}\text { Accélération dans les } \\
\text { axes } X, Y \text { et } Z\end{array}$ & $\begin{array}{c}\text { Accélération dans les } \\
\text { axes X, Y et } Z \\
\text { Rotation autour des axes } \\
\text { X, Y et Z }\end{array}$ \\
\hline 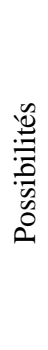 & $\begin{array}{c}\text { Mouvement de } \\
\text { l'accélération du point } \\
\text { où est fixé le capteur, } \\
\text { suivant le seul axe Z en } \\
\text { l'absence de rotation } \\
\text { Mesure de l'inclinaison } \\
\text { par rapport à la verticale } \\
\text { en l'absence de } \\
\text { mouvement }\end{array}$ & $\begin{array}{c}\text { Mesure de } \\
\text { l'accélération du point } \\
\text { où est fixé le capteur } \\
\text { suivant les trois axes X, } \\
\text { Y, Z du capteur } \\
\text { Mesure de l'inclinaison } \\
\text { dans les trois axes en } \\
\text { l'absence de } \\
\text { mouvement }\end{array}$ & $\begin{array}{c}\text { Mesures de } \\
\text { l'accélération du point } \\
\text { où est fixé le capteur } \\
\text { dans les trois axes X, Y, } \\
\text { Z du capteur } \\
\text { Mesure d'inclinaison } \\
\text { dans les trois axes }\end{array}$ \\
\hline 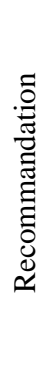 & $\begin{array}{c}\text { Mesure sur guide barre } \\
\text { obligatoire } \\
\text { L’axe de } \\
\text { l’accéléromètre doit être } \\
\text { impérativement } \\
\text { parallèle, tout au long } \\
\text { du geste, à l'un des axes } \\
\text { du référentiel galiléen } \\
\text { Aucune rotation }\end{array}$ & $\begin{array}{l}\text { Mesure sur une barre } \\
\text { L’orientation du } \\
\text { référentiel de } \\
\text { l’accéléromètre doit être } \\
\text { connue, tout au long du } \\
\text { geste par rapport au } \\
\text { référentiel galiléen } \\
\text { Mouvements libre ave } \\
\text { Peu de rotation }\end{array}$ & $\begin{array}{l}\text { Mouvement libres } \\
\text { L’orientation du } \\
\text { référentiel de } \\
\text { l'accéléromètre est } \\
\text { connue dans le } \\
\text { référentiel galiléen à } \\
\text { l’aide des gyroscopes }\end{array}$ \\
\hline
\end{tabular}

Avant de choisir un accéléromètre ou une centrale inertielle, il est très important de prendre en considération ses caractéristiques techniques qui peuvent être très différentes d'un capteur à l'autre. On n'utilisera pas le même accéléromètre pour mesurer les vibrations subies par la raquette de tennis lors d'une frappe de balle que pour mesurer l'accélération subie par une hanche lors de la marche. La sensibilité et la bande passante de l'accéléromètre sont deux caractéristiques importantes. Dans le cadre de l'analyse des mouvements humains la fréquence d'enregistrement sera différente en fonction de l'objectif. Pour mesurer le temps passé immobile ou bien en mouvement tout au long d'une journée dans le but par exemple de définir le degré de sédentarité on utilisera plutôt un accéléromètre avec une fréquence comprise entre 25 et $60 \mathrm{~Hz}$ (Bouten et al., 1997 ; Chen et al., 2005 ; Cleland et al., 2013 ; Kavanagh et al., 2008). Pour la mesure des accélérations d'un point sur le corps lors d'un saut ou d'une course ou bien encore d'une barre de musculation on 
utilisera des fréquences allant de 100 à $1000 \mathrm{~Hz}$ (Choukou et al., 2014 ; Crewther et al., 2011 ; Jidovtseff et al., 2006 ; Sato et al., 2009). Plus le mouvement est dynamique et plus la fréquence d'enregistrement devra être élevée si on veut obtenir une image précise du mouvement analysé sans erreur lors des procédures de calcul. La sensibilité est également très importante. Alors que certains capteurs peuvent mesurer des accélérations allant jusqu'à $100 \mathrm{~g}$, la sensibilité des accéléromètres utilisés pour l'analyse des mouvements sportifs est généralement comprise entre $\pm 2 \mathrm{~g}$ et $\pm 10 \mathrm{~g}$ (Kavanagh et al., 2008). Un accéléromètre $\pm 2 \mathrm{~g}$ possèdera une meilleure sensibilité pour les mouvements plus lents comme la marche mais ne pourra pas être utilisé si le mouvement comprend des accélérations dépassant les $2 \mathrm{~g}$. A l'inverse un accéléromètre 5 ou $10 \mathrm{~g}$ sera mieux adapté aux mouvements les plus dynamiques comme la course ou les sauts mais pourrait manquer de sensibilité lorsque le mouvement subit des accélérations très faibles. Pour obtenir une sensibilité accrue sur un répertoire gestuel et dynamique plus large, les ingénieurs proposent maintenant des systèmes miniatures qui intègrent plusieurs accéléromètres aux caractéristiques complémentaires.

De par sa petite taille, son faible poids et son prix attractif, l'accéléromètre apparaît comme une technologie intéressante et accessible dans l'analyse des mouvements humains (Callaway et al., 2009 ; Mayagoitia et al., 2002b), l'analyse du niveau d'activité physique (Bouten et al., 1997 ; Chen et al., 2005 ; Cleland et al., 2013 ; Ward et al., 2005) mais aussi l'exploration de la performance musculaire (Bampouras et al., 2013 ; Caruso et al., 2012 ; Comstock et al., 2011 ; Jidovtseff et al., 2008 ; Thompson et al., 1999).

Si les premières études biomécaniques utilisant l'accélérométrie apparaissent dans les années 1980 (Chu et al., 1986), c'est seulement à la fin des années 1990 que l'on a utilisé les accéléromètres pour mesurer la performance musculaires dans des exercices de musculation (Thompson et al., 1999 ; Bemben et al., 1999). Au début, ce sont des capteurs uniaxiaux qui ont été fixés sur des machines de musculation afin de mesurer l'accélération d'une charge de masse constante dans un mouvement de développé couché et dans un mouvement de presse.

\section{3. Évaluation et analyse de la performance musculaire par accélérométrie ${ }^{1}$}

Pour évaluer et analyser la performance lors de mouvements de musculation, il est intéressant de mesurer le déplacement, la vitesse ainsi que l'accélération de la charge. A cette fin, différents capteurs physiques peuvent être utilisés comme les accéléromètres, mais aussi les capteurs de déplacement (CD) ou encore les plateformes de force (PFF). Cependant, la modélisation du mouvement devra être déterminée en fonction du choix de l'outil. Les deux systèmes que sont le capteur de déplacement et l'accéléromètre sont facilement transportables et peuvent s'utiliser à la fois en laboratoire et en salle de musculation. Les CD sont munis d'un fil

1. Chapitre réalisé avec la participation du professeur Patrick Lacouture. 
(figure 5) qui s’attache à la barre mobilisée et qui s’enroule et se déroule au gré du mouvement. Les accéléromètres généralement se placent directement sur la charge mobilisée ou sur le sujet.
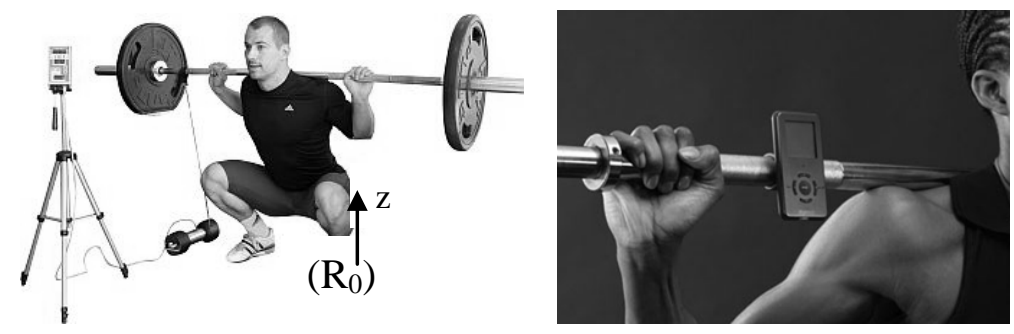

Figure 5. Présentation d'un capteur de déplacement (Tendo Units $\left.{ }^{\circledR}\right)$ et d'un accélérométre (Myotest Pro $\left.{ }^{\circledR}\right)$. C'est dans le référentiel galiléen $R_{0}$ que doit s'effectuer l'ensemble des mesures et calculs. Il faut donc s'assurer que les capteurs de déplacement ou d'accélérométrie mesurent bien suivant l'axe vertical $Z$

Lorsque le capteur (de déplacement ou accéléromètre) est fixé au centre de gravité de la barre on obtient la vitesse du centre de gravité (CG) de la barre, soit en dérivant le déplacement, soit en intégrant l'accélération, conformément aux relations suivantes :

$V_{Z}(t)=\frac{d Z(t)}{d t}$ avec $V_{Z}(t)$, la vitesse du CG de la barre et $Z(t)$, le déplacement vertical du CG de la barre mesuré par le CD ;

$\mathrm{Ou}$

$$
V_{Z}(t)=\int_{t_{0}}^{t} A_{Z}(t) d t+V_{Z}\left(t_{0}\right) \text {, avec } A_{Z}(t) \text { l'accélération du CG de la barre }
$$

mesurée dans le référentiel du capteur accéléromètre qui doit rester parallèle au référentiel galiléen tout au long du geste. $\boldsymbol{V}_{\mathbf{Z}}\left(\boldsymbol{t}_{\mathbf{0}}\right)$ la vitesse initiale du CG de la barre, nulle si la barre est immobile à cet instant initial.

Par ailleurs, en appliquant le principe fondamental de la dynamique sur le système barre, nous obtenons l'expression de la force verticale d'action du sujet sur la barre, soit :

$$
F_{Z}(t)=M_{b}\left[A_{Z}(t)-g\right]
$$

Que représente alors le produit force*vitesse, soit : $\boldsymbol{F}_{\mathbf{Z}}(\boldsymbol{t}) \boldsymbol{V}_{\mathbf{Z}}(\boldsymbol{t})$ ?

En multipliant l'équation (2), par $\boldsymbol{V}_{\boldsymbol{Z}}(\boldsymbol{t})$, nous obtenons : 


$$
F_{Z}(t) V_{Z}(t)=M_{b}\left[A_{Z}(t)-g\right] V_{Z}(t)
$$

En remplaçant dans (3), $\boldsymbol{V}_{\mathbf{Z}}(\boldsymbol{t})$ et $\boldsymbol{A}_{\mathbf{Z}}(\boldsymbol{t})$ par leur expression respective telle que $V_{z}(t)=\frac{d Z(t)}{d t}$ et que $A_{z}(t)=\frac{d V_{z}(t)}{d t}$, l'équation (3) s'écrit :

$$
F_{Z}(t) V_{Z}(t)=M_{b} \frac{d V_{Z}(t)}{d t} V_{Z}(t)-M_{b} g \frac{d Z(t)}{d t}
$$

Or :

$$
\begin{aligned}
\frac{d V_{Z}(t)}{d t} V_{Z}(t) & =\frac{1}{2} \frac{d V^{2}{ }_{Z}(t)}{d t} \\
\text { D'où } F_{Z}(t) V_{Z}(t) & =\frac{1}{2} M_{b} \frac{d V^{2}{ }_{Z}(t)}{d t}-M_{b} g \frac{d Z(t)}{d t} \\
= & \frac{d}{d t}\left[\frac{1}{2} M_{b} V^{2}(t)-M_{b} g Z(t)\right] \\
& F_{Z}(t) V_{Z}(t)=\frac{d}{d t}\left(E_{c e x t}+E_{p}\right)
\end{aligned}
$$

Avec $\boldsymbol{E}_{\boldsymbol{c e x t}}$ représentant l'énergie cinétique externe de la barre et $\boldsymbol{E} \boldsymbol{p}$, son énergie potentielle.

L’équation (4) s’écrit également :

$$
P_{F_{Z}}=F_{Z}(t) V_{Z}(t)=\frac{d E_{m e x t}}{d t}
$$

avec $\boldsymbol{E}_{\boldsymbol{m} \boldsymbol{m e x t}}$, l’énergie mécanique externe de la barre.

Ainsi, le produit force*vitesse $\boldsymbol{P}_{\boldsymbol{F}_{\boldsymbol{z}}}$ exprime alors la puissance de la force verticale résultante exercée par l'athlète sur la barre à différents niveaux de charge ; nous obtenons des informations plus complètes que la simple et traditionnelle détermination de la charge maximale qui peut être soulevée une seule fois (1RM). À l'aide de ces mesures, il est possible de caractériser la capacité de l'athlète à accélérer une charge donnée (figure 6).

Il est indispensable de comprendre que l'utilisation de capteurs physiques positionnés sur la charge constitue une approche indirecte de l'analyse de mouvement basée sur le calcul d'une puissance ou d'un travail d'une force externe appliquée à la barre. Ainsi, l'accéléromètre permet d'accéder à la force externe 
résultat de l'action du sujet sur la barre, mais ne permet pas de connaitre les forces développées au niveau de chaque articulation nécessaires pour réaliser ce soulevé de charge. Il en est de même pour les calculs de vitesse, de force et de puissance obtenues qui nous informent uniquement sur les conséquences extérieures du mouvement (impact sur la charge mobilisée), mais ne nous informe en rien sur l'implication individuelle des groupes musculaires et articulations dans l’établissement de la vitesse et de la puissance extérieures.

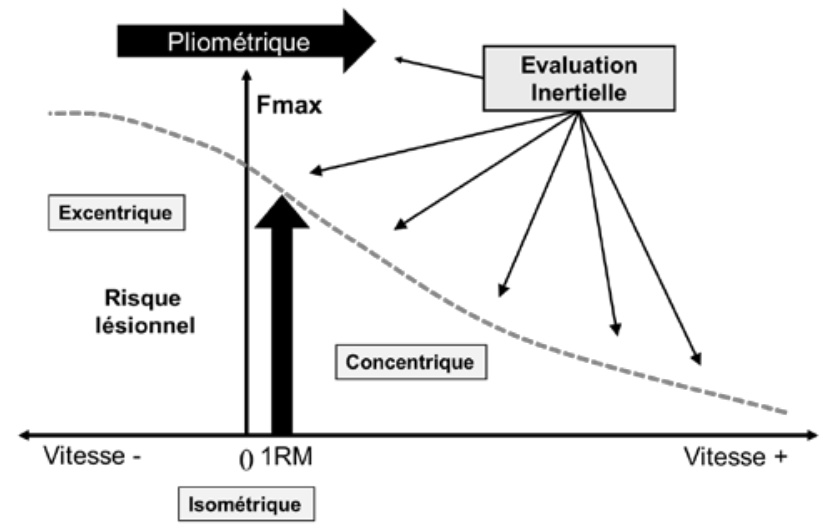

Figure 6. Limite du 1RM et apport de l'évaluation inertielle dans l'exploration de la relation force vitesse

Pour bien comprendre cela, appliquons le théorème de l'énergie cinétique au système athlète. Nous pouvons écrire :

$$
\Delta E_{c \text { totale }}=W_{F} \text { int }+W_{F e x t}
$$

Avec : $\boldsymbol{\Delta \boldsymbol { E } _ { \boldsymbol { c } } \text { totale }}$, la variation de l’énergie cinétique totale, égale à :

_ $\Delta E_{\text {c totale }}=\Delta E_{c \text { int }}+\Delta E_{\text {cext }}$ où $\Delta E_{\text {c int }}$ est la variation de l'énergie cinétique interne, qui prend en compte les énergies cinétiques de translation et de rotation des segments corporels ; $\boldsymbol{\Delta E}_{\boldsymbol{c e x t}}$ est la variation de l'énergie cinétique du CG de l'athlète.

_ $\boldsymbol{W}_{\boldsymbol{F}}$ int, le travail des forces internes,

_ $\boldsymbol{W}_{\boldsymbol{F e x t}}$, le travail des forces externes que sont le poids de l'athlète, la force de réaction au sol, et la force d'action de la barre sur les mains de l'athlète.

Une fois l'équation (6) développée, nous obtenons : 


$$
W_{F \text { int }}=\Delta E_{\text {int }}+\Delta E_{c e x t}+\Delta E_{p}+W_{F z}
$$

Ou bien exprimée en termes de puissance, l’équation (8) devient :

$$
P_{F \text { int }}=\frac{d E_{\text {cint }}}{d t}+\frac{d E_{c e x t}}{d t}+\frac{d E_{p}}{d t}+P_{F z}
$$

Ainsi, la puissance de la force résultante verticale d'action $\boldsymbol{P}_{\mathbf{F z}}$ serait égale à la puissance des forces internes $\boldsymbol{P}_{\boldsymbol{F}}$ int , à la seule condition que la somme des termes $\frac{d E_{\text {c int }}}{d t}+\frac{d E_{c e x t}}{d t}+\frac{d E_{p}}{d t}$ soit nulle, ce qui est loin d'être le cas puisque pour soulever la charge, l'athlète doit se mettre en mouvement sous l'action des muscles actionneurs !

En conséquence on ne peut pas assimiler la puissance $\boldsymbol{P}_{\boldsymbol{F z}}$ à la puissance des forces internes $\boldsymbol{P}_{\boldsymbol{F}}$ int , par ailleurs égale à la somme des puissances articulaires $\boldsymbol{P}_{\boldsymbol{i}}$ soit :

\section{$\boldsymbol{P}_{\boldsymbol{F}}$ int $=\sum \boldsymbol{P i}$. Notons que ces puissances $\boldsymbol{P}_{\boldsymbol{i}}$ sous-estiment la i articulations}

puissance développée par chacun des muscles sollicités. L'introduction d'un modèle musculaire est alors nécessaire. Cette problématique est actuellement un enjeu scientifique dans les laboratoires de biomécanique qui étudie le mouvement.

La mesure de la vitesse et de la puissance à partir d'un accéléromètre permet donc théoriquement une exploration globale des profils musculaires (figure 7).
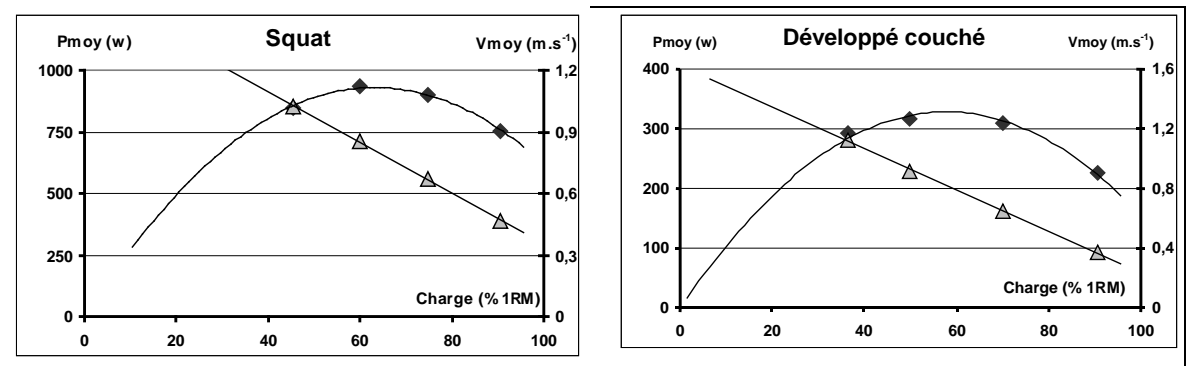

Figure 7. Évolution de la vitesse verticale moyenne (Vmoy) du CG de la barre et de la puissance verticale moyenne en fonction de la charge relative (en \% du 1RM) lors de l'évaluation musculaire en squat et en développé couché (Jidovtseff et al., 2006)

L’accéléromètre placé sur la barre ou sur la charge mobilisée, permet de mesurer la force externe, la vitesse et la puissance à différents niveaux de charge. Les profils musculaires ainsi définis permettent d'établir, pour un mouvement de musculation donné, les relations charge-vitesse $(\mathrm{Ch}-\mathrm{V})$ et charge-puissance $(\mathrm{Ch}-\mathrm{P})$ utiles pour 
l'entraineur. Pour être complet, un profil devra explorer les qualités musculaires à charge légère (pour explorer les qualités de vitesse), à charge moyenne (pour explorer les qualités de puissance) et à charge élevée (pour explorer les qualités de force) (Jidovtseff et al., 2008). Ce concept d'évaluation musculaire offre évidemment bien plus d'informations que le seul 1RM.

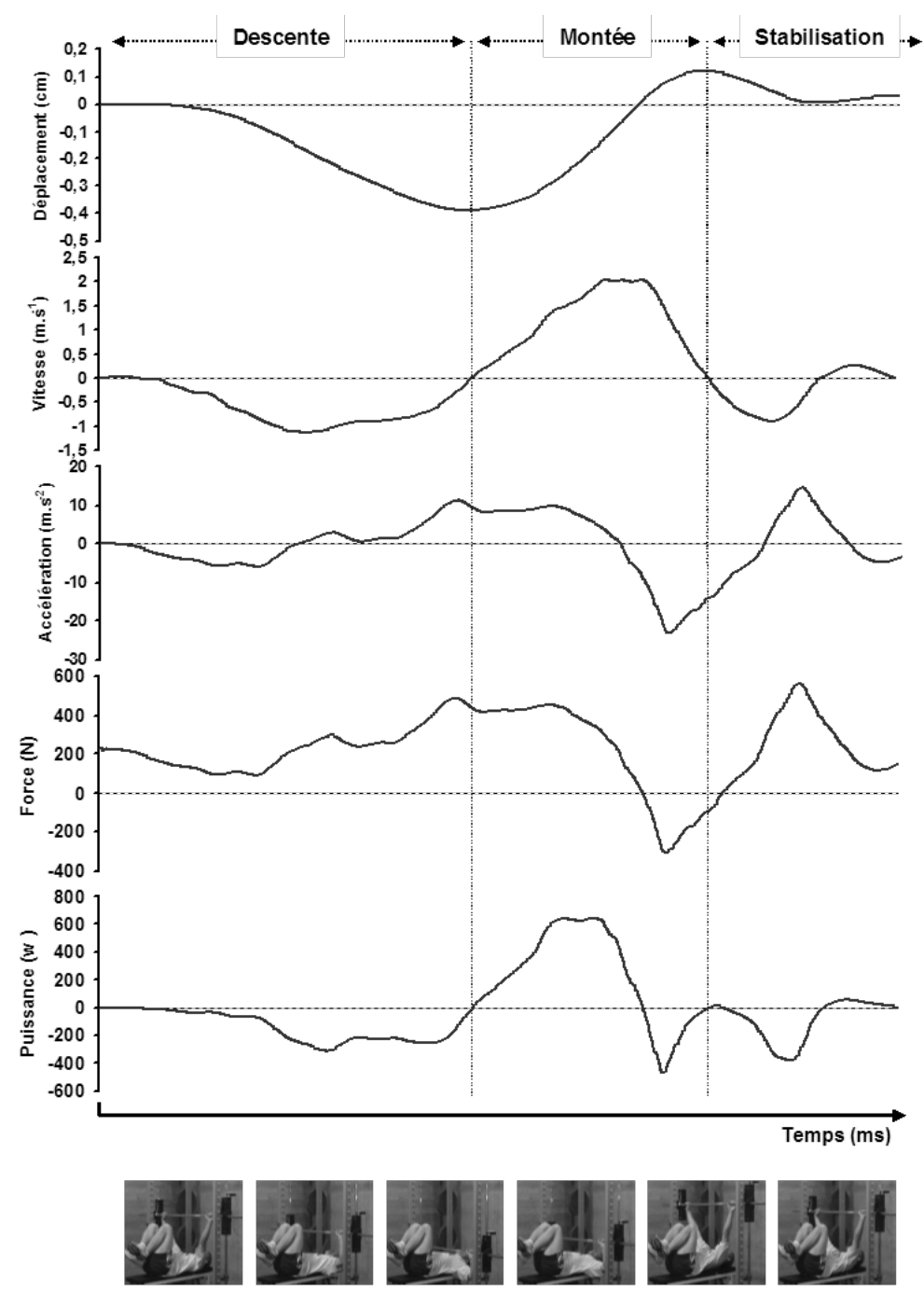

Figure 8. Courbes du déplacement vertical $\mathbf{Z}(\boldsymbol{t})$, de la vitesse $\boldsymbol{V}_{\mathbf{Z}}(\boldsymbol{t})$, de l'accélération $\boldsymbol{A}_{\mathbf{Z}}(\boldsymbol{t})$, de la force verticale $\boldsymbol{F}_{\mathbf{Z}}(\boldsymbol{t})$ et de la puissance $\boldsymbol{P}_{\mathbf{F}_{\mathbf{Z}}}(\boldsymbol{t})$ en fonction du temps au cours d'un développé couché complet (Jidovtseff, 2006) 
La mesure de la vitesse développée à des charges sous maximales est également utilisée pour estimer le 1RM (Jidovtseff et al., 2012; Bosquet et al., 2010; Jidovtseff et al., 2011). Cette approche est réellement intéressante car en mesurant les performances musculaires à une charge légère (20 à $50 \%$ du $1 \mathrm{RM})$ et à une charge plus lourde mais sous maximale (70-80\%1RM) on obtient non seulement une bonne estimation du 1RM sans passer par des charges extrêmement lourdes, mais en plus on a un bon aperçu des qualités de vitesse et de puissance musculaire du sportif (Jidovtseff et al., 2011).

L'analyse cinétique des courbes accélération-temps, force-temps, vitesse-temps, puissance-temps et déplacement-temps permet une analyse biomécanique des mouvements de musculation (figure 8).

L'évolution au fil des répétitions des paramètres physiques extérieurs permet également une exploration précise de la résistance à la fatigue musculaire et de l'endurance musculaire (figure 9) (Harris et al. 2010 ; Jidovtseff, 2006).

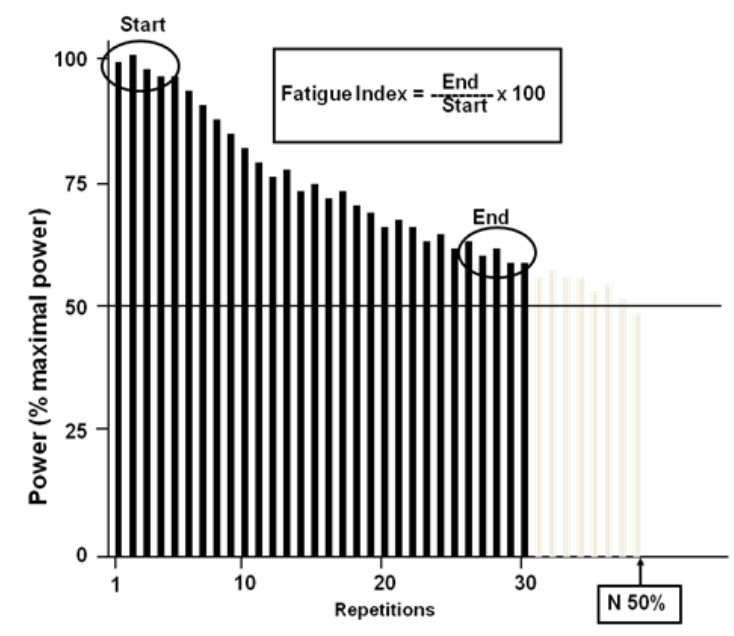

Figure 9. Représentation schématique d'un protocole de résistance à la fatigue (Harris et al., 2010)

\section{Conditions optimales d'utilisation des accéléromètres}

\subsection{Validité}

Les études de validité se sont attachées à comparer les performances obtenues à partir d'accéléromètres à celles obtenues à partir d'autres systèmes de référence. Malheureusement, les études qui se sont penchées sur la validité des accéléromètres lors de l'évaluation musculaire restent peu nombreuses et présentent des protocoles assez différents. L'évaluation de la performance en salle de musculation s'avère 
complexe car elle dépend de nombreuses variables dont les trois principales sont le mouvement, l'individu, et le système de mesure. Pour connaître les qualités intrinsèques d'un système de mesure, il est intéressant de standardiser au maximum les autres variables. Ainsi, pour s'affranchir de la variabilité liée à l'individu, une simulation de mouvement a été réalisée sur un guide barre vertical (Smith machine) afin de comparer un accéléromètre uniaxial à un CD (Jidovtseff, 2006). L'accélération mesurée par le $\mathrm{CD}$ est très légèrement supérieure à celle obtenue par l'accéléromètre $(0,2 \%)$. Cette différence est cependant systématique et significative. Pour la vitesse et la puissance cette différence monte à $5 \%(\mathrm{p}<0,001)$ et pour le déplacement elle atteint $10 \%(\mathrm{P}<0,001)$. Dans le cadre de cette étude quatre hypothèses peuvent expliquer ces résultats : une erreur systématique de mesure de l'accéléromètre ou du $\mathrm{CD}$, une calibration imparfaite, une détection du mouvement retardé par l'accéléromètre ou encore une légère inclinaison du capteur. L'erreur systématique très faible $(0,2 \%)$ n'explique pas à elle toute seule une différence qui monte à $10 \%$ pour le déplacement. Dans le cas présent la détection du mouvement retardée par l'accéléromètre apparaît probablement comme le facteur le plus influent car la moindre erreur de départ est amplifiée après chaque calcul mathématique. C'est ce que nous observons ici avec une erreur qui s'accroît à chaque intégrale. La calibration peut se faire de manière manuelle ou de manière automatique avant les débuts des tests. Pour les accéléromètres uniaxiaux il est très important que le capteur soit parfaitement positionné à l'horizontale au moment de cette mesure sous peine d'engendrer une erreur systématique qui risque de s'amplifier à chaque intégrale. Cette simulation de mouvement montre à quel point l'accéléromètre est un outil sensible et à quel point une erreur initiale peut avoir des répercussions importantes sur les mesures de vitesse, de puissance et de déplacement.

Les deux études qui ont étudié la validité des accéléromètres dans un mouvement strictement vertical se sont attardées sur le développé couché. La première étude utilise également un accéléromètre uniaxial pour mesurer l'accélération, la vitesse, la puissance et le déplacement lors d'un DC réalisé à $60 \%$ du 1RM (Thompson et al., 1999). Les auteurs comparent les mesures maximales à celles obtenues à partir d'une caméra et les valeurs moyennes à celle obtenues à partir de cellules photoélectriques. Les corrélations entre les systèmes de mesure apparaissent élevées $(0,93-0,98)$. La corrélation la plus faible est toutefois observée pour le déplacement. Des corrélations élevées indiquent le lien de proportionnalité entre les deux systèmes de mesure, mais ne nous informe en aucun cas si les mesures sont les mêmes. La comparaison des moyennes révèle que l'accéléromètre offre des valeurs systématiquement inférieures à celles obtenues à partir de la caméra, rejoignant les observations de la simulation de mouvement.

La seconde étude compare à quatre niveaux de charge $(35,50,70$ et $95 \% 1 \mathrm{RM})$ le Myotest de première génération (accéléromètre uniaxial) à un système optimisé qui combine un CD et un accéléromètre (Jidovtseff et al., 2008). Ces conditions très standardisées permettent d'observer entre les deux systèmes des corrélations extrêmement élevées pour la force maximale (Fmax) à tous les niveaux de charge 
(0,992-0,996). L'étude révèle que pour la puissance maximale (Pmax) et la vitesse maximale (Vmax) les corrélations restent très élevées aux trois premières charges $(0,973-0,998)$ mais se réduisent à la charge la plus élevée $(0,83-8,85)$. A cette charge proche du maximum (95\% 1RM), la vitesse et la puissance mesurées par l'accélérométrie sont respectivement de 36 et 39 \% inférieures aux valeurs mesurées par le système de référence. Aux charges lourdes, la lenteur initiale rend la détection du début du mouvement extrêmement difficile pour les accéléromètres, compromettant la validité des mesures de vitesse, de puissance et de déplacement. Dans le cas de cette étude, la limite de validité du Myotest est clairement dépassée à $95 \%$ du 1RM. La détection du vrai début de mouvement reste complexe avec un accéléromètre car il est extrêmement difficile de faire la distinction entre une augmentation du signal liée au début du mouvement et une augmentation brutale du signal liée à un choc sur la barre. La figure 10 montre que les mesures qui correspondent à $95 \%$ du $1 \mathrm{RM}$ s'écartent significativement de la régression linéaire établie pour les autres charges entre le Myotest et le système contrôle.

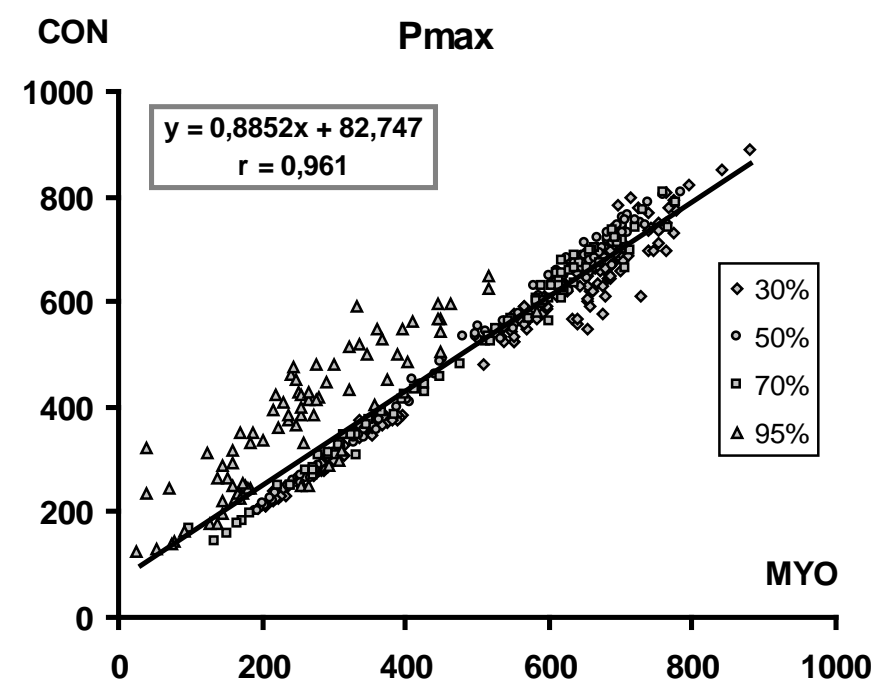

Figure 10. Corrélation entre les valeurs de Pmax obtenues par le Myotest (MYO) et celles obtenues par le système contrôle (CON) à 35, 50, 70 et $95 \%$ du 1RM

Plusieurs études récentes ont étudié la validité de l'accélérométrie dans des conditions de mouvement non guidées, plus proches de la réalité de terrain (Bampouras et al., 2013 ; Comstock et al., 2011 ; Crewther et al., 2011 ; McMaster et al., 2013 ; Sato et al., 2009). Les résultats ne semblent pas montrer l'existence d'un biais plus important lorsque le mouvement n'est pas guidé. Par contre, les corrélations entre l'accéléromètre et le système de référence apparaissent généralement plus faibles. 
Tableau 2. Validité de l'accélérométrie dans l'évaluation de la performance musculaire

\begin{tabular}{|c|c|c|c|c|c|c|}
\hline Auteurs & $\begin{array}{l}\text { Instru- } \\
\text { ments }\end{array}$ & $\begin{array}{l}\text { Mouve- } \\
\text { ments }\end{array}$ & $\begin{array}{l}\text { Condi- } \\
\text { tions de } \\
\text { tests }\end{array}$ & Paramètre & Test & Score \\
\hline $\begin{array}{l}\text { Bampouras } \\
\text { et al., } 2013\end{array}$ & $\begin{array}{c}A C C \text { vs } \\
P F F\end{array}$ & Squat jump & $\begin{array}{l}\text { Mouvem } \\
\text { ent libre }\end{array}$ & Fmax & $\begin{array}{c}R \\
\text { Biais }\end{array}$ & $\begin{array}{l}0,815(p=0,001) \\
F_{P F F}=0,81 F_{A C C} b\end{array}$ \\
\hline $\begin{array}{l}\text { Comstock } \\
\text { et al, } 2011\end{array}$ & $\begin{array}{c}A C C \text { vs } \\
P F F+ \\
C D\end{array}$ & $\begin{array}{c}\text { SJ } 30 \% \\
1 R M \\
S Q 1 R M \\
D C 30 \% \\
1 R M \\
D C 1 R M\end{array}$ & $\begin{array}{c}\text { Mouve- } \\
\text { ment } \\
\text { libre }\end{array}$ & $\begin{array}{l}\text { Pmax } \\
\text { Fmax } \\
\text { Pmax } \\
\text { Fmax }\end{array}$ & $\begin{array}{c}R^{2} \\
R^{2} \\
R^{2} \\
R^{2} \\
\text { Biais }\end{array}$ & $\begin{array}{c}0,56-0,82 \\
0,73-0,97 \\
0,86-0,98 \\
0,92-0,97 \\
\text { Pas de mesure }\end{array}$ \\
\hline $\begin{array}{l}\text { Crewther } \\
\text { et al. } \\
\text { (2011) }\end{array}$ & $\begin{array}{c}A C C \text { vs } \\
P F F\end{array}$ & $\begin{array}{c}S J 20-40-60- \\
80 \mathrm{~kg}\end{array}$ & $\begin{array}{c}\text { Mouve- } \\
\text { ment } \\
\text { libre }\end{array}$ & $\begin{array}{l}\text { Fmax } \\
\text { Pmax }\end{array}$ & $\begin{array}{c}R \\
\text { Biais } \\
R \\
\text { Biais }\end{array}$ & $\begin{array}{c}0,85-0,97 \\
+7 N \grave{a}+271 N^{a} \\
0,66-0,90 \\
-180 W \grave{a} \\
+141 W\end{array}$ \\
\hline $\begin{array}{c}\text { Jidovtseff } \\
\text { et al. } \\
(2006)\end{array}$ & $\begin{array}{c}A c c \text { vs } \\
C D\end{array}$ & $\begin{array}{c}\text { Simulation } \\
\text { de } \\
\text { mouvement } \\
\text { concentrique }\end{array}$ & $\begin{array}{l}\text { Mouve- } \\
\text { ment } \\
\text { guidé }\end{array}$ & $\begin{array}{l}\text { Courbe A } \\
\text { Courbe V } \\
\text { Courbe P } \\
\text { Courbe D }\end{array}$ & $\begin{array}{l}\% \\
\% \\
\% \\
\%\end{array}$ & $\begin{array}{c}-0,2 \%^{b} \\
-5 \%^{b} \\
-5 \%^{b} \\
-10 \%^{b}\end{array}$ \\
\hline $\begin{array}{c}\text { Jidovtseff } \\
\text { et al. } \\
(2008)\end{array}$ & $\begin{array}{c}A c c \text { vs } \\
A c c+C \\
D\end{array}$ & $\begin{array}{c}D C 30,50, \\
70, \\
95 \% 1 R M\end{array}$ & $\begin{array}{l}\text { Mouve- } \\
\text { ment } \\
\text { guidé }\end{array}$ & $\begin{array}{l}\text { Fmax } \\
\text { Biais } \\
\text { Pmax } \\
\text { Biais } \\
\text { Vmax } \\
\text { Biais }\end{array}$ & $\begin{array}{l}R \\
\% \\
R \\
\% \\
R \\
\%\end{array}$ & $\begin{array}{c}0,992-0,996 \\
-1 \% a ̀-6 \% \\
0,85-0,99 \\
-1 \% \grave{a}-39 \%^{b} \\
0,83-0,998 \\
-2 \% a ̀ ~-36 \%^{b}\end{array}$ \\
\hline $\begin{array}{l}\text { Sato et al. } \\
\quad(2009)\end{array}$ & $\begin{array}{c}\text { Acc vs } \\
\text { Caméra } \\
H V\end{array}$ & $\begin{array}{c}\text { Tirage } \\
\text { vertical } \\
40 \mathrm{~kg}\end{array}$ & $\begin{array}{c}\text { Mouve- } \\
\text { ment } \\
\text { libre }\end{array}$ & Courbe A & $R$ & 0,94-0,99 \\
\hline $\begin{array}{c}\text { Thompson } \\
\text { et } \\
\text { Bemben, } \\
\text { (1999) }\end{array}$ & $\begin{array}{l}\text { Acc vs } \\
\text { Caméra }\end{array}$ & $\begin{array}{c}D C \\
60 \% 1 R M\end{array}$ & $\begin{array}{c}\text { Mouvem } \\
\text { ent } \\
\text { guidé }\end{array}$ & $\begin{array}{l}\text { Pmoy } \\
\text { Biais } \\
\text { Vmoy } \\
\text { Biais } \\
\text { Dmax } \\
\text { Biais }\end{array}$ & $\begin{array}{l}R \\
\% \\
R \\
\% \\
R \\
\%\end{array}$ & $\begin{array}{c}0,95 \\
-14 \%^{a} \\
0,98 \\
-13 \%^{a} \\
0,93 \\
-9 \%^{a}\end{array}$ \\
\hline
\end{tabular}

Significativité des biais : $a=p<0,05 ; b=p<0,001$ ACC : accéléromètre, PFF : plateforme de force, $C D$ : caméra digitale.

Plusieurs études ont comparé les mesures du Myotest Pro avec celles d'une plateforme de force lors de squat jumps sautés avec ou sans charge (Bampouras et al., 2013 ; Choukou et al., 2014 ; Crewther et al., 2011 ; McMaster et al., 2013). Dans l'étude de Bampouras et al. (2013), le squat jump est réalisé avec un bâton sur les épaules, sans autre charge additionnelle. Seule la force maximale (Fmax) est étudiée. Si la corrélation entre les deux systèmes apparaît satisfaisante $(\mathrm{r}=0,815)$, l'accélération offre systématiquement des mesures supérieures à la PFF. Crewther 
qui a réalisé des squats sautés avec $20,40,60$ et $80 \mathrm{~kg}$ observe la même tendance à la charge la plus faible (Crewther et al., 2011). La différence entre l'accéléromètre et la PFF diminue avec l'augmentation de la charge et devient insignifiante à $80 \mathrm{~kg}$. Cette variation s'explique par les différences entre le mouvement du centre de masse (CM) mesuré par la PFF et celui de la barre sur laquelle l'accéléromètre est fixé. Il a été démontré que l'instrument de mesure utilisé et son emplacement influence la mesure de la force et de la puissance lors de squats jumps (Cormie et al., 2007 ; Crewther et al., 2011 ; Hori et al., 2006 ; McMaster et al., 2013).

En effet, le corps n'est pas un système rigide et le mouvement mesuré au niveau de la barre par un accéléromètre n'est pas équivalent au mouvement du système entier. Dans l'étude de Crewther et al. (2011), lorsque la charge augmente, la position du centre de masse (CM) se rapproche de la barre, expliquant partiellement la diminution des différences observées entre les deux systèmes. À charge légère, la flexion plus importante du tronc pourrait aussi intervenir dans la différence observée. Un redressement brutal du tronc impacterait beaucoup plus la cinétique de la barre sur laquelle l'accéléromètre est placé que le CM mesuré par la plateforme de force.

Une étude récente montre que le positionnement de l'accéléromètre influence les résultats (McMaster et al., 2013). Cette étude qui compare un Myotest à une plateforme de force confirme que la mesure de la force est surestimée lorsque le Myotest est positionné sur une barre à hauteur des épaules. Les auteurs ne constatent par contre aucune différence avec la plateforme de force lorsque le Myotest est fixé à la hanche. Il est donc fondamental de connaître la position du capteur afin de donner une bonne interprétation aux résultats. Ces résultats sont confirmés par Choukou et al. (2014). Les deux études apparaissent par contre contradictoires en ce qui concerne la puissance et la vitesse.

Plusieurs études révèlent qu’à partir d’un accéléromètre, la validité de la puissance et de la vitesse reste inférieure à celle de la force mesurée sur plateforme de force (Crewther et al., 2011 ; Choukou et al., 2014 ; Comstock et al., 2011 ; Jidovtseff et al., 2008 ; McMaster et al., 2013). Comstock qui a comparé le Myotest Pro à un système de référence combinant une PFF avec un CD pour les mouvements de squat et de développé couché montre que les coefficients de détermination de Fmax sont systématiquement plus élevés que ceux de Pmax. Les auteurs malheureusement ne précisent pas s'il existe une différence entre les deux systèmes de mesure. Ils révèlent par contre que la validité de l'accéléromètre semble meilleure lors du mouvement de développé couché, comparativement au squat. Lors des sauts dynamiques, la validité de Pmax et de Vmax apparaît clairement insuffisante (Choukou et al., 2014 ; McMaster et al., 2013). Il y a lors de ces mouvements une rotation du tronc en avant que l'on ne retrouve pas dans le DC. Cette rotation associée à un déplacement horizontal de l'accéléromètre altère inévitablement les mesures verticales du déplacement et de la vitesse de la barre. En effet, en l'absence d'un gyroscope il est impossible, dans ce contexte, de détecter la part d'accélération liée à la rotation, au déplacement vertical, au déplacement 
horizontal et à la gravité. Toute rotation excessive durant un mouvement de musculation compromet inévitablement la validité des mesures obtenues à partir de l'accéléromètre, que celui-ci soit fixé sur le corps ou sur la barre. En réalisant les mouvements sur un guide barre on limite tout mouvement horizontal et toute rotation et on améliore significativement la validité des données.

Si le squat et le DC restent les mouvements les plus étudiés certains auteurs se sont penchés sur d'autres mouvements de musculation. Sato et al. (2009) ont comparés les résultats d'un accéléromètre triaxial avec ceux d'une caméra haute vitesse lors d'un mouvement de tirage haltérophile vertical (Sato et al., 2009). Leur analyse se limite aux courbes d'accélération qui apparaissent très comparables pour les deux systèmes ( $\mathrm{R}$ de 0,94 à 0,99$)$. L'auteur précise dans son travail que les mouvements haltérophiles complets (arraché et épaulé jeté) n’ont pu être mesurés à cause des mouvements de rotation subis par la barre en fin de mouvement. Une fois de plus les rotations posent problème avec les accéléromètres non accompagnés de gyroscopes. L’auteur précise également que les accéléromètres ne peuvent être utilisés dans les mouvements haltérophiles sans utiliser une protection adéquate des capteurs. En effet après avoir été soulevée, la barre est généralement jetée sur le plateau. Le choc très important pourrait occasionner des dégâts irréversibles au capteur si celui-ci n’est pas protégé par une gaine adaptée (figure 11).

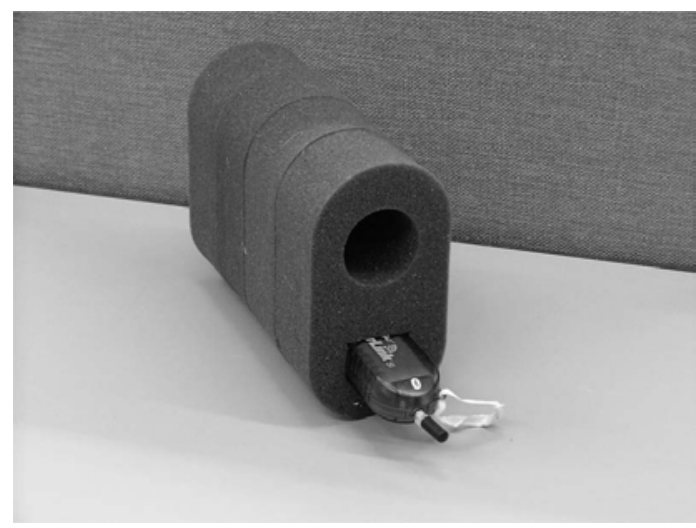

Figure 11. Montage réalisé pour protéger l'accéléromètre lors des mouvements haltérophiles (Sato et al., 2009)

Les études de validité nous informent sur l'exactitude des données mesurées et sur l'interchangeabilité des instruments. Il apparaît que les mesures obtenues par plateforme de force ne peuvent être directement comparées à celles observées avec les accéléromètres. Ce constat résulte d'approches biomécaniques différentes : cinétique pour la plateforme de force (PFF) et cinématique pour l'accéléromètre. D'après l'étude de Gomez-Piriz (Gomez-Piriz et al., 2013), il semblerait qu'il n’y ait pas d’interchangeabilité entre accéléromètre et capteur de déplacement, qui, 
pourtant, reposent tous les deux sur la cinématique. Il observe pour la vitesse, la puissance et la force des mesures supérieures avec l'accéléromètre. Ces résultats sont en contradiction avec ceux obtenus par Jidovtseff et al. (2008) qui présentent des valeurs plus faibles avec un accéléromètre comparées à celles obtenues avec un capteur de déplacement. Plusieurs différences de protocoles pourraient justifier ces observations. Dans la première étude le DC est réalisé avec une barre libre alors que dans la seconde il est réalisé sur un guide barre. Tout d'abord, dans un mouvement non guidé, une rotation de l'accéléromètre peut survenir, augmentant l'amplitude du signal et donc les résultats. Cette éventuelle rotation n’a aucun impact sur la mesure du capteur de déplacement. Ensuite, les mouvements réalisés avec barre libre ne sont pas toujours symétriques et il est fréquent d'observer une dominance latérale qui peut interférer sur le maintien parfait de la barre à l'horizontale durant la poussée. Il suffit que l'accéléromètre soit placé plus à l'extérieur que le capteur de déplacement pour subir des variations plus importantes du mouvement. De plus le fait d'attacher le câble du capteur de déplacement d'un côté de la barre induit une résistance supplémentaire qui favorise certainement l'asymétrie. Idéalement, le système de mesure devrait se positionner au centre de la barre. Pour des raisons techniques évidentes, c'est très difficile et on essayera de rapprocher les capteurs le plus possible de cette position. Ces problèmes n'apparaissent pas lorsque le mouvement est guidé dans un axe strictement vertical. Il faut noter que les résultats de l'étude de Gomez-Pirriz (Gomez-Piriz et al., 2013) sont basés sur l'observation de seulement trois sujets et à un seul niveau de charge, déforçant la signification des différences observées. Cette étude semble clairement démontrer une plus grande variabilité des données issues de l'accéléromètre en comparaison avec celles issues du capteur de déplacement. Ces observations sont quant à elles conformes aux études de reproductibilités relevées dans la littérature.

\subsection{Reproductibilité}

Les études de reproductibilité des accéléromètres présentent des résultats assez variables qui semblent dépendre des conditions de mouvement (guidé versus non guidé), du mouvement en lui-même, de la charge, du capteur et de la motivation des sujets. Les tests utilisés dans les études ne sont pas systématiquement les mêmes ce qui complique l'analyse. Les conditions d'évaluation les plus standardisées devraient théoriquement s'accompagner d'une meilleure reproductibilité. L'analyse de la littérature montre effectivement que pour la mesure de l'accélération et de la force, la reproductibilité des accéléromètres est excellente lorsque le mouvement est guidé (Jidovtseff, 2006 ; Jidovtseff et al., 2008 ; Thompson et al., 1999). Les coefficients de corrélation intraclasse sont proches de l'unité (Thompson et al., 1999) et les coefficients de variation sont inférieurs à $3 \%$ (Jidovtseff, 2006 ; Jidovtseff et al., 2008). Lorsque l'on s'attarde à la vitesse, à la puissance et au déplacement, la reproductibilité apparaît plus faible (Cabrera et al., 2009; Jidovtseff et al., 2008). Tout comme pour la validité, la reproductibilité des mesures obtenues à partir des accéléromètres semble se détériorer à chaque opération mathématique. 
Une simulation de mouvement montre cette augmentation des coefficients de variation $(\mathrm{CV})$ : 2,1\% pour l'accélération ; $5 \%$ pour la vitesse ; 6,1\% pour la puissance et 7,1\% pour le déplacement (Jidovtseff, 2006). La faible reproductibilité du déplacement à partir d'un accéléromètre a clairement été mise en évidence dans les études sur les sauts (Choukou et al., 2014).

Tableau 3. Reproductibilité de l'accélérométrie dans l'évaluation de la performance musculaire

\begin{tabular}{|c|c|c|c|c|c|}
\hline Auteurs & Mouvements & $\begin{array}{c}\text { Conditions } \\
\text { de tests }\end{array}$ & Paramètre & CCI & CV (\%) \\
\hline \multirow{3}{*}{$\begin{array}{l}\text { Bampouras } \\
\text { et al., } 2013\end{array}$} & \multirow{3}{*}{ Squat jump } & \multirow{3}{*}{$\begin{array}{l}\text { Mouvement } \\
\text { libre }\end{array}$} & Fmax & 0,90 & 2,1 \\
\hline & & & Pmax & 0,80 & 2,3 \\
\hline & & & Vmax & 0,84 & 3,2 \\
\hline \multirow{2}{*}{$\begin{array}{l}\text { Cabrera } \\
\text { et al., } 2009\end{array}$} & \multirow{2}{*}{$D C$} & \multirow{2}{*}{$\begin{array}{l}\text { Mouvement } \\
\text { guidé }\end{array}$} & Pmax & 0,80 & \\
\hline & & & Pmoy & 0,93 & \\
\hline \multirow{3}{*}{$\begin{array}{c}\text { Caruso et } \\
\text { al, } 2012\end{array}$} & \multirow{3}{*}{$\begin{array}{c}\text { SQ front } 55- \\
65-75- \\
80 \% 1 R M\end{array}$} & \multirow{3}{*}{$\begin{array}{l}\text { Mouvement } \\
\text { libre }\end{array}$} & Fmax & $0,73-0,85$ & $4,7-7,8$ \\
\hline & & & Pmax & $0,10-0,97$ & $17,8-36,5$ \\
\hline & & & Vmax & $0,14-0,86$ & $20,4-34$ \\
\hline $\begin{array}{l}\text { Comstock } \\
\text { et al., } 2011\end{array}$ & $S Q$ et $D C$ & $\begin{array}{l}\text { Mouvement } \\
\text { libre }\end{array}$ & $\begin{array}{l}\text { Pmax, } \\
\text { Fmax, }\end{array}$ & $\geq 0,96$ & \\
\hline \multirow{2}{*}{$\begin{array}{l}\text { Crewther } \\
\text { et al. } 2011\end{array}$} & \multirow{2}{*}{$\begin{array}{c}S J 20-40-60- \\
80 \mathrm{~kg}\end{array}$} & \multirow{2}{*}{$\begin{array}{l}\text { Mouvement } \\
\text { libre }\end{array}$} & Fmax & & 2,6 \\
\hline & & & Pmax & & 3,3 \\
\hline \multirow{4}{*}{$\begin{array}{l}\text { Jidovtseff } \\
\text { et al, } 2006\end{array}$} & \multirow{4}{*}{$\begin{array}{c}\text { Simulation } \\
\text { de } \\
\text { mouvement } \\
\text { concentrique }\end{array}$} & \multirow{4}{*}{$\begin{array}{l}\text { Mouvement } \\
\text { guidé }\end{array}$} & Courbe A & & 2,1 \\
\hline & & & Courbe V & & 5 \\
\hline & & & Courbe $P$ & & 6,1 \\
\hline & & & Courbe D & & 7,1 \\
\hline \multirow{3}{*}{$\begin{array}{l}\text { Jidovtseff } \\
\text { et al., } 2008\end{array}$} & \multirow{3}{*}{$\begin{array}{c}D C 30,50, \\
70,95 \% 1 R M\end{array}$} & \multirow{3}{*}{$\begin{array}{l}\text { Mouvement } \\
\text { guidé }\end{array}$} & Fmax & & $1,6-2,8$ \\
\hline & & & Pmax & & $4,9-25,3$ \\
\hline & & & $V \max$ & & $3,2-24,4$ \\
\hline $\begin{array}{l}\text { Koshida et } \\
\text { al., } 2008 .\end{array}$ & $D C 50 \% 1 R M$ & $\begin{array}{l}\text { Mouvement } \\
\text { libre sur } \\
\text { banc ou sur } \\
\text { ballon. }\end{array}$ & Amax & $0,94-0,96$ & \\
\hline
\end{tabular}

Dans des conditions de mouvement guidé, les études montrent la bonne reproductibilité de la vitesse et de la puissance obtenues à partir d'un accéléromètre aux charges sous maximales. Les valeurs observées (Jidovtseff, et al., 2008 ; Thompson et al., 1999) sont dans la plupart des cas comparables aux mesures de reproductibilité observées avec des capteurs de déplacement (Bosco et al., 1995 ; Harris et al., 2010 ; Jidovtseff et al., 2006) ou des plateformes de force (Hori et al., 2006 ; Rahmani et al., 2000), confirmant la pertinence de l’accélérométrie dans 
l'évaluation de la performance musculaire. La reproductibilité de la vitesse et de la puissance serait par contre problématique à charge élevée. Une étude qui a mesuré avec un Myotest Fmax, Vmax et Pmax à 30, 50, 70 et $95 \%$ du 1RM montre qu'aux trois premières charges, les $\mathrm{CV}$ sont excellents pour tous les paramètres (CV entre 2 et $8 \%$ ) (Jidovtseff et al., 2008). A $95 \%$ du 1RM les CV de Vmax et Pmax s'élèvent respectivement à $24,4 \%$ et à $25,3 \%$. Cette charge l'accélération est tellement faible qu'il est difficile de détecter le vrai début du mouvement. Une erreur de détection de ce début de mouvement se répercute en cascade sur le calcul de la vitesse, de la puissance et encore plus sur celui du déplacement.

En condition non guidée la reproductibilité des mesures obtenues par les accéléromètres apparaît de manière générale plus faible (voir tableau 3), tout en restant acceptable dans la plupart des études (Bampouras et al., 2013)(Cabrera et al., 2009; Comstock et al., 2011 ; Crewther et al., 2011 ; Koshida et al., 2008). Lors des sauts, les mesures de Vmax, Pmax et Dmax apparaissent peu reproductibles (Choukou et al., 2014 ; McMaster et al., 2013). Tous insistent sur le respect d'une série de critères. L'accéléromètre durant le mouvement doit rester le plus près possible de l'axe vertical. Les rotations doivent être évitées dans la mesure du possible. Que ce soit en développé couché ou en squat, prendre une barre libre d'un rack et la redéposer après l'effort provoque une rotation problématique pour l'accélérométrie. L'enregistrement du signal doit donc être initié après cette manipulation et stoppé avant de replacer la barre sur le rack. Certains constructeurs comme Myotest demandent de respecter une période d'immobilisation d'une seconde avant et après chaque mouvement. Cette période qui garantit un signal plat de l'accélération avant et après l'effort est utilisée pour améliorer la qualité des données et compenser les éventuelles dérives liées aux intégrales. Son non-respect réduit inévitablement la qualité des données.

L'étude de Caruso et al. (2012) mesure à plusieurs charges Fmax, Pmax et Vmax en squat front non guidé avec un Myotest chez des footballeurs américains (Caruso et al., 2012). Si les résultats observés pour Fmax semblent satisfaisants (ICC $\geq 0,73$ et $\mathrm{CV}<8 \%$ ) ils restent néanmoins inférieurs aux valeurs observées dans les autres études. Les indices de reproductibilité observés pour Pmax et Vmax apparaissent insuffisants et particulièrement à la charge la plus légère ou les CV s'élèvent respectivement à 36,5 et $34 \%$. Cette plus faible reproductibilité pourrait s'expliquer par la difficulté motrice de réaliser un effort dynamique maximum avec des charges faibles sans pouvoir réaliser un saut. Le contrôle difficile de la fin de mouvement a été évoqué pour expliquer une plus faible reproductibilité des performances à charge légère lors d'un squat non sauté (Jidovtseff et al., 2006). Il est également possible qu'aux charges plus légères, la rotation avant du tronc soit plus importante, ce qui inévitablement altèrerait la qualité des données. Les auteurs avancent aussi l'hypothèse d'une plus faible motivation des sujets à cette charge légère (Caruso et al., 2012). Il est évident que l'évaluateur doit faire en sorte que la motivation et la collaboration des sujets restent maximales à chaque mesure. La reproductibilité de l'évaluation inertielle dépend du mouvement, de la charge, du système de mesure, 
mais aussi des sujets. Pour que le test reflète au mieux ses capacités musculaires, il est indispensable de s'assurer que le sujet :

- maîtrise le mouvement d'évaluation ;

- soit en pleine possession de ses moyens ;

- collabore en se donnant à son maximum à chaque évaluation.

Le non-respect de ces consignes altèrerait inévitablement la qualité de l’évaluation musculaire réalisée.

\section{Applications pour la détermination d'un « profil musculaire » par accélérométrie}

Un intérêt majeur des accéléromètres est de permettre la réalisation de profils musculaires pour des mouvements de musculation réalisés à l'entraînement comme le squat, le développé couché ou encore la presse. Plusieurs études ont déjà utilisé les accéléromètres dans cette perspective (Caruso et al., 2012 ; Crewther et al., 2011; Jidovtseff et al., 2008 ; Sleivert et al., 2004). D’après les études de validité et de reproductibilité il faudra cependant éviter les charges très élevées qui s'accompagnent d'un début de mouvement lent (Jidovtseff et al., 2008). Bien qu'aucune étude n’offre une limite de validité précise, nous déconseillons d’utiliser des charges supérieures à $90 \%$ du 1RM. Aux charges faibles, les difficultés résident dans l'existence de mouvement et de rotations parasites ainsi que la difficulté de réaliser un effort réellement maximum si celui-ci doit être maitrisé dans sa phase finale. L’évaluation devrait idéalement être réalisée sur un guide barre et avec une projection ou une autoprotection qui permet au sujet d'exprimer sans retenue ses capacités maximales.

Ces profils musculaires présentent de nombreuses applications : identifier les caractéristiques musculaires d'une population; situer un sportif dans un groupe; mettre en relation avec la performance fonctionnelle et optimaliser l'entraînement en définissant avec plus de précision les zone de travail et en vérifiant son efficacité (Bosco et al., 1995 ; Harris et al., 2010 ; Jidovtseff, 2006 ; Jidovtseff et al., 2008, 2009 ; Miller, 1997 ; Sleivert et al., 2004).

L’accélérométrie peut être utilisée durant la séance d’entraînement afin d'informer l'athlète directement sur sa performance. Ce feedback peut constituer un élément motivationnel ou un élément de contrôle. Un système comme le Myotest dispose d'un écran sur lequel les principaux résultats sont immédiatement affichés après l'effort. Sans devoir mobiliser un ordinateur, il est possible de mesurer la vitesse et la puissance à une charge donnée. La facilité du système autorise une utilisation régulière qui permet un suivi objectif l'entraînement. Un entraîneur peut par exemple, en début de chaque séance, réaliser des mesures à 40kg en développé couché. L'évolution de la vitesse au cours de l'année offrira à l'entraîneur une information objective sur l'état de forme de son athlète. Cette même approche peut aussi être utilisée pour s’assurer que le sportif se donne réellement à son maximum 
lors des entrainements. En effet, lorsque l'on travaille la vitesse ou la puissance musculaire, on soulève généralement des charges sous-maximales avec un nombre limité de répétitions. Cet entraînement n'est réellement efficace que si l'athlète, à chaque répétition, essaye de développer la vitesse la plus élevée possible (Cronin et al., 2005 ; Miller, 1997). Actuellement, l'accélérométrie constitue certainement la solution la plus pratique pour réaliser ce contrôle. Les profils musculaires, qui eux, ne sont pas réalisés de manière fréquentes étant donné le temps qu'ils nécessitent, peuvent être utilisés de manière judicieuse pour déterminer à chaque niveau de charge la vitesse qui devrait être atteint par le sportif. A titre d'exemple, la feuille de résultat présentée dans la figure 12 montre le profil charge-vitesse-puissance d'un sportif pour un exercice de presse. Une modélisation de la relation charge-vitesse permet de définir les zones d'entraînement et de calculer les vitesses que ce sportif devrait atteindre avec un Myotest à chaque niveau de charge. Ces valeurs peuvent être utilisées par l'entraîneur afin de vérifier l'évolution de son sportif ou encore sa bonne collaboration durant l'entraînement. Les profils sont également utilisés pour déterminer des zones d'entrainement. L'accélérométrie peut être aussi utilisée pour objectiver l'apparition de la fatigue. Si l'objectif de l'entraînement est hyperqualitatif, la mesure par accélérométrie permet de détecter le moment où la performance commence à baisser. Ainsi, l'étude de Sato et al. (2009) proposent d'utiliser un accéléromètre dans l'entraînement haltérophile afin d'une part, d'analyser la courbe d'accélération dans la phase de tirage, mais aussi afin de détecter la survenue de la fatigue (Sato et al., 2009).

Il n'est pas toujours possible ni utile d'établir un profil musculaire et l'évaluation de la performance peut se limiter à une ou deux charges. Le choix d'une charge moyenne (entre 30 et $70 \%$ du $1 \mathrm{RM}$ ) permet généralement d'explorer les qualités de puissance musculaire (Cormie et al., 2007; Harris et al., 2010 ; Jidovtseff et al., 2009 ; Miller, 1997). Ce type d'approche a été utilisé avec succès afin d'étudier les effets de l'entraînement musculaire (Jidovtseff et al., 2014). Le Myotest, par exemple, a été utilisé chez des joueuses de handball non professionnelles afin d'objectiver l'efficacité de deux courtes séances de musculation sur la puissance des membres inférieurs, la puissance des bras et la détente verticale (Jidovtseff et al., 2014). Plusieurs études ont utilisé un accéléromètre afin d'explorer la potentiation musculaire lors du travail par contraste de charge en développé couché (Liossis et al., 2013, Cabrera et al., 2009 ; Morio et al., 2011 ; Ruben et al., 2010). Un accéléromètre uniaxial a été utilisé afin d'étudier l'évolution de la puissance des membres inférieurs (presse) et la puissance des bras (développé couché) en fonction de l'âge (Bemben et al., 1999). 


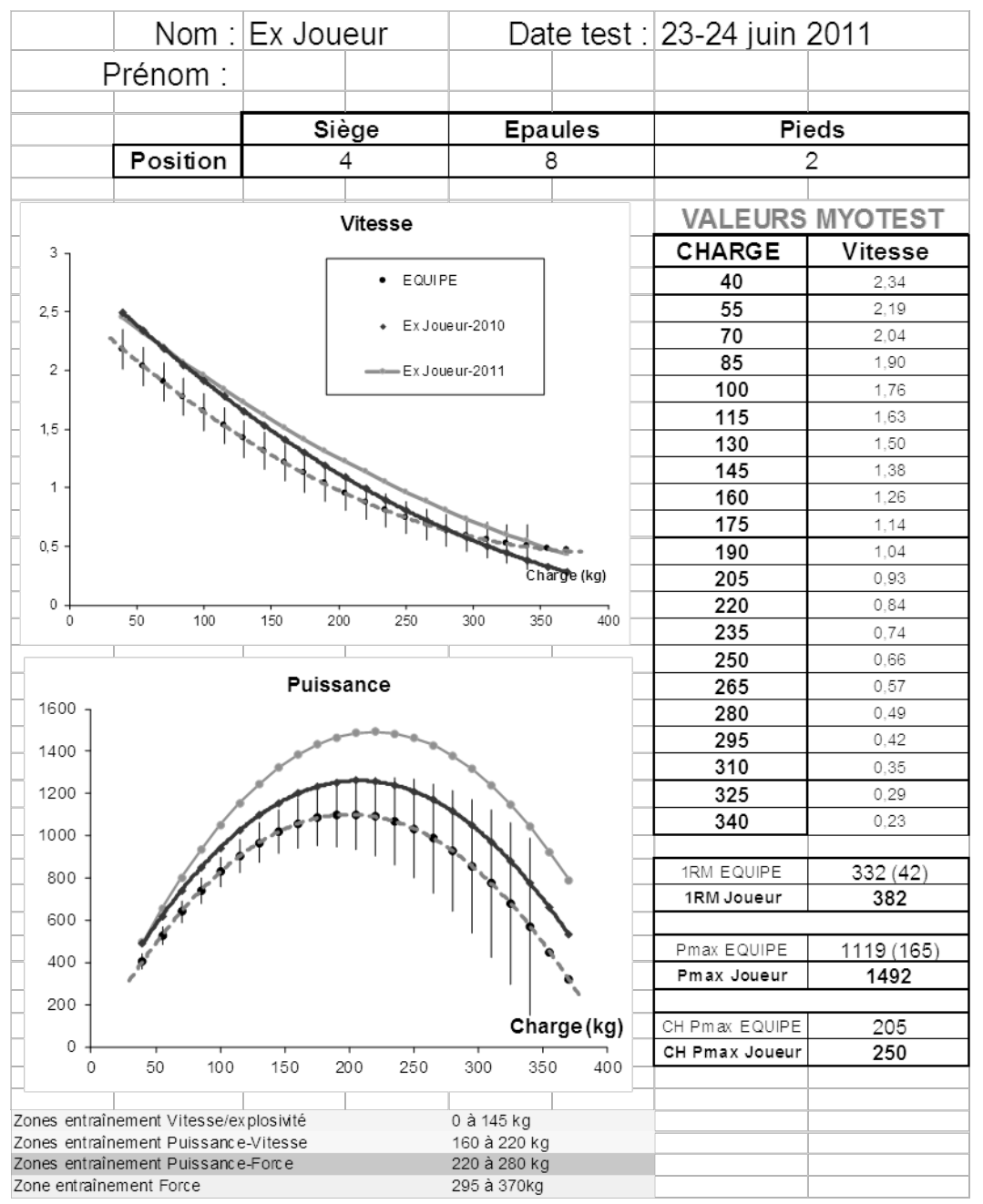

Figure 12. Feuille de résultat présentant pour un sportif de haut niveau son profil charge-vitesse-puissance en comparaison avec les valeurs moyennes de l'équipe, ses zones d'entraînement et les vitesses qu'il devrait atteindre avec un Myotest à chaque niveau de charge

L'estimation du 1RM à partir de charges sous maximales fait l'objet de nombreuses recherches (Mayhew et al., 2008; Pereira et al., 2003). L’accélérométrie offrirait de nouvelles perspectives. Il a été récemment démontré que la mesure de la vitesse à des charges sous maximale permet une bonne estimation du 1RM (Bosquet et al., 2010 ; Jidovtseff et al., 2012 ; Jidovtseff et al., 2011). La seule étude ayant utilisé un accéléromètre montre que la qualité prédictive de la méthode semble 
dépendre du mouvement. Satisfaisante pour le DC et pour la presse elle est apparue impossible sur une machine Leg Curl (Jidovtseff et al., 2012). Dans le même ordre d'idée, un accéléromètre triaxial qui se porte au poignet comme une montre a été utilisé afin d'estimer le 1RM (Hannula et al., 2008 ; Rontu et al., 2010). Le principe consiste à mesurer l'accélération maximale (Amax) durant la phase concentrique d'un développé couché réalisé à intensité maximale avec une charge donnée. Les auteurs qui ont exploré à différentes charges la relation entre le 1RM et Amax proposent une équation qui possèderait un excellent pouvoir prédictif (erreur d'estimation $<5 \%$ ) lorsque la charge utilisée est comprise entre 70 et $80 \%$ du $1 \mathrm{RM}$ (équation (9)).

$1 R M=1,0187 \cdot\left(A_{\max } / 9,91\right) \cdot m+6,9836$
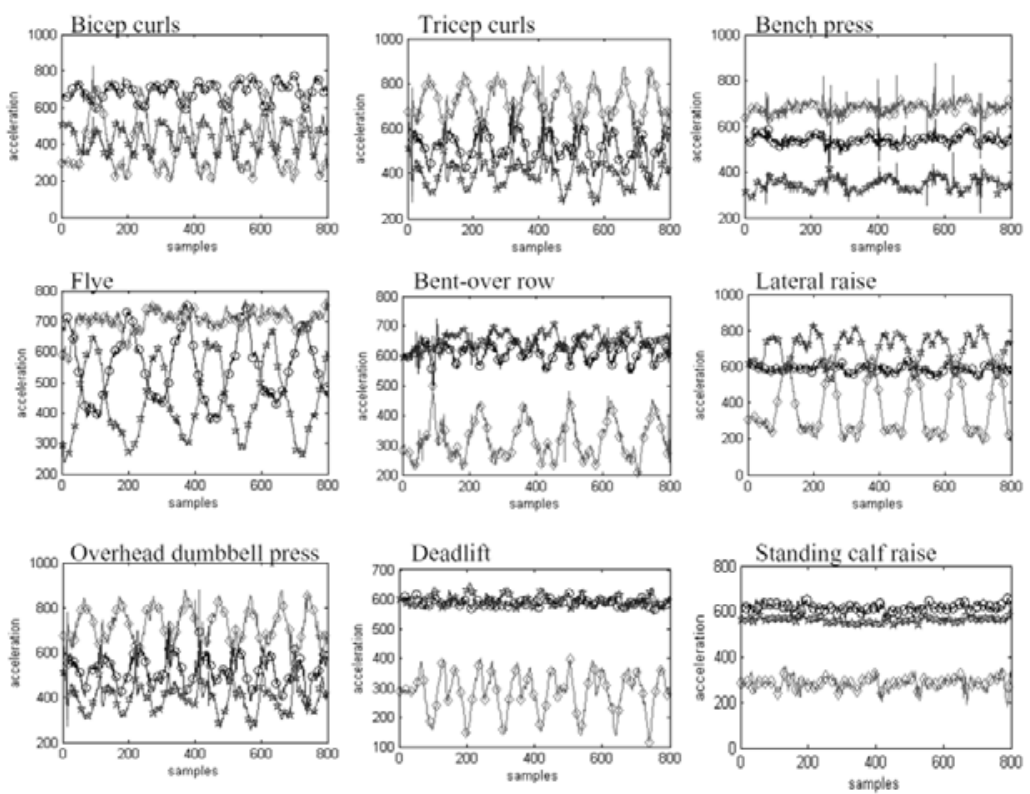

Figure 13. Pattern des accélérations mesurées dans les axes $x, y$ et $\mathrm{z}$ pour neuf exercices de musculation (Chang et al., 2007)

L'accélérométrie peut être utilisée dans l'analyse biomécanique des efforts musculaires, des mouvements de musculation ou encore dans l'analyse du matériel. Des accéléromètres ont ainsi été utilisés pour évaluer les efforts excentriques retrouvés dans les actions frénatrices, pour étudier les mouvements d'inversion retrouvés dans les efforts pliométriques et pour explorer les actions propulsives (Hasegawa et al., 2011; Morio et al., 2011). Ainsi par exemple, une équipe de chercheurs (Vingren et al., 2011) a utilisé le Myotest Pro afin de quantifier les 
diminutions de force, de vitesse et de puissance induite par les systèmes de contre balanciers que l'on retrouve sur certains guides barres.

Finalement l'accélérométrie peut aussi être utilisée en salle de musculation à des fins de reconnaissance de mouvement. En plaçant un accéléromètre à la hanche et un autre sur le poignet, Chang et al. (2007) ont réussi à reconnaître 9 exercices de musculation avec une précision qui s'élève à $95 \%$ de réussite. Des algorithmes permettent de reconnaître les patterns spécifiques de chaque mouvement (figure 13) tout en assurant un comptage précis des répétitions ( $<5 \%$ d'erreur). Cette application ouvre la perspective d'une supervision électronique qui peut s'avérer intéressante pour, par exemple, motiver des sujets à avoir une activité physique mais aussi pour quantifier le niveau de sédentarité.

\section{Conclusions et perspectives}

Les accéléromètres sont des outils de mesure de petite taille, légers et économiques qui permettent d'évaluer la performance musculaire sans perturber le mouvement fonctionnel. Les études de validité et de reproductibilité, ainsi que la compréhension technique des différents accéléromètres, ont permis de définir les critères à respecter et le cadre d'utilisation.

Les accéléromètres uniaxiaux doivent être bien fixés sur la charge mobilisée et ne se déplacer que dans un seul axe dont l'orientation doit être connue tout au long du mouvement étudié par rapport au référentiel galiléen. Les accéléromètres triaxiaux, malgré les prétentions de certains commerciaux, offrent à peine plus de libertés. L'accéléromètre ne doit pas obligatoirement être placé dans l'axe de mesure mais le mouvement devra subir le moins de rotation possible car sans gyroscope, il est impossible de déterminer la part d'accélération liée à une éventuelle inclinaison et la part d'accélération liée aux mouvements dynamiques. Pour garantir la qualité des données, il est recommandé de placer l'accéléromètre sur une charge qui est mobilisée dans le seul axe vertical.

Les systèmes actuels ne permettent pas de garantir la fiabilité des résultats lorsque le mouvement est très lent, à charge élevée.

Ces conditions optimales, dépendant des caractéristiques technologiques des accéléromètres actuels, pourraient être remises en question avec les avancées technologiques. Les centrales inertielles qui combinent accélérométrie et gyroscope offrent des perspectives futures évidentes. Grace aux mesures gyroscopiques il est envisageable d'obtenir des mesures de qualité dans des mouvements de musculation avec rotation comme le squat ou encore les mouvements haltérophiles. Si certaines limites apparaissent lors des mouvements très dynamiques les perspectives apparaissent très encourageantes au vu des rapides évolutions technologiques. 


\section{Bibliographie}

Bampouras T. M., Relph N. S., Orme D., Esformes J. I. (2013). Validity and reliability of the Myotest Pro wireless accelerometer in squat jumps. Isokinetics and Exercise Science, vol. 21, n 2 , p. 101-105.

Bemben M. G., McCalip G. A. (1999). Strength and power relationships as a function of age. Journal of Strength and Conditioning Research, vol. 13, n 4, p. 330-338.

Bosco C., Belli A., Astrua M., Tihanyi J., Pozzo R., Kellis, S., Tsarpela, O., Foti, C., Manno R., Tranquilli C. (1995). A dynamometer for evaluation of dynamic muscle work. European Journal Of Applied Physiology And Occupational Physiology, vol. 70, $\mathrm{n}^{\circ}$ 5, p. 379-386.

Bosquet L., Porta-Benache J., Blais J. (2010). Validity of a commercial linear encoder to estimate bench press $1 \mathrm{RM}$ from the force-velocity relationship. Journal of Sports Science and Medicine, 9, p. 459-463.

Bouten C.V., Koekkoek K. T., Verduin M., Kodde R., Janssen J. D. (1997). A triaxial accelerometer and portable data processing unit for the assessment of daily physical activity. Biomedical Engineering, IEEE Transactions on, vol. 44, n 3, p. 136-147.

Cabrera C. A., Morales J., Greer F., Pettitt R. W. (2009). Exercise Bouts at Three Different Intensities Fail to Potentiate Concentric Power. International Journal of Exercise Science, vol. $2, \mathrm{n}^{\circ} 1$.

Callaway A. J., Cobb J. E., Jones I. (2009). A comparison of video and accelerometer based approaches applied to performance monitoring in swimming. International Journal of Sports Science and Coaching, vol. 4, n 1, p. 139-153.

Caruso J. F., Olson N. M., Taylor S. T., McLagan J. R., Shepherd C. M., Borgsmiller J. A., Riner R.R., Gilliland L., Grisewold S. (2012). Front squat data reproducibility collected with a triple-axis accelerometer. The Journal of Strength \& Conditioning Research, vol. 26, n 1 , p. 40-46.

Chang K.H., Chen M.Y., Canny J. (2007). Tracking free weight exercises. Ubiquitous Computing. J Krumm et al. (Eds.) Springer, p. 19-37.

Chen K. Y., Bassett D. R. (2005). The technology of accelerometry-based activity monitors: Current and future. Medicine and science in sports and exercise, vol. 37, $\mathrm{n}^{\circ} 11$, S490S500.

Choukou M., Laffaye G., Taiar R. (2014). Reliability and validity of an accele-rometric system for assessing vertical jumping performance. Biol. Sport, 31, p. 55-62.

Chu M. L., Yazdani-Ardakani S., Gradisar I. A., Askew M. J. (1986). An in vitro simulation study of impulsive force transmission along the lower skeletal extremity. Journal of Biomechanics, vol. 19, n 12, p. 979-987.

Cleland I., Kikhia B., Nugent C., Boytsov A., Hallberg J., Synnes K., Finlay D. (2013). Optimal Placement of Accelerometers for the Detection of Everyday Activities. Sensors, vol. 13, n 7 , p. 9183-9200.

Comstock B. A., Solomon-Hill G., Flanagan S. D., Earp J. E., Luk H.-Y., Dobbins K. A., Hatfield D. L. (2011). Validity of the Myotest ${ }^{\circledR}$ in measuring force and power production 
in the squat and bench press. The Journal of Strength \& Conditioning Research, vol. 25, $\mathrm{n}^{\circ}$ 8, p. 2293-2297.

Cormie P., McBride J. M., McCaulley G. O. (2007). Validation of power measurement techniques in dynamic lower body resistance exercises. Journal of Applied Biomechanics, 23, p. 103-118.

Crewther B., Kilduff L., Cunningham D., Cook C., Owen N., Yang G. (2011). Validating two systems for estimating force and power. International Journal of Sports Medicine, vol. 32, $\mathrm{n}^{\circ} 4,254$.

Cronin J. B., Sleivert, G. (2005). Challenges in understanding the influence of maximal power training on improving athletic performance. Sports Medicine, vol. 35, n 3, p. 213-234.

Frosio I., Pedersini F., Alberto Borghese N. (2009). Autocalibration of MEMS Accelerometers. Instrumentation and Measurement, IEEE Transactions on, vol. 58, $\mathrm{n}^{\circ} 6$, p. 2034-2041.

Gomez-Piriz P. T., Sanchez E. T., Manrique D. C., Gonzalez E. P. (2013). Reliability and comparability of the accelerometer and the linear position measuring device in resistance training. The Journal of Strength \& Conditioning Research, vol. 27, n 6, p. 1664-1670.

Hannula M., Hirvikoski A. (2008). Validation of individual calibration procedure in prediction of one repetition maximum in bench press. 14th Nordic-Baltic Conference on Biomedical Engineering and Medical Physics, A. Katashev, Y. Dekhtyar, J. Spigulis (Eds.), vol. 20, p. 115-118.

Harris N. K., Cronin J., Taylor K. L., Boris J., Sheppard J. (2010). Understanding Position transducer technology for strength and conditioning practitioners. Strength and Conditioning Journal, 32, $\mathrm{n}^{\circ}$ 4, p. 66-79.

Hasegawa H., Yamauchi T., Kawasaki T., Adachi T., Yamashita M., Nakashima, N. (2011). Effects of plyometric training using a portable self-coaching system on running performance and biomechanical variables in jump exercises. The Journal of Strength \& Conditioning Research, 25.

Hori N., Newton R. U., Nosaka K., McGuigan M. R. (2006). Comparison of different methods of determining power output in weightlifting exercises. Strength and Conditioning Journal, vol. 28, $\mathrm{n}^{\circ}$ 2, p. 34-40.

Intille S., Lester J., Sallis J., Duncan G. (2012). New horizons in sensor development. Medicine and science in sports and exercise, vol. 44, $\mathrm{n}^{\circ} 1$, Suppl 1, p. S24-31.

Jidovtseff B. (2006). Mise au point d'un dynamomètre de la puissance musculaire. University of Liege, Liege.

Jidovtseff B., Frère P., Theunissen C. (2014). Influence de la musculation sur la performance physique en handball féminin amateur.

Jidovtseff B., Cronin J., Crielaard J.-M., Villaret J., Harris N. (2012). 1RM prediction and load-velocity relationship. Paper presented at the Abstract book of 8th International Conference on Strength Training.

Jidovtseff B., Harris N. K., Crielaard J. M., Cronin J. B. (2011). Using the load-velocity relationship for 1RM prediction. $J$ Strength Cond Res, vol. 25, n 1, p. 267-270. 
Jidovtseff B., Bruls O., Tubez F., Monfort L., Harris, N., Cronin J. (2010). Using inertia measurement unit (IMU) for exercise analysis. Paper presented at the 7th International conference on strength training-abstract book.

Jidovtseff B., Quièvre J., Hanon C., Crielaard J.-M. (2009). Les profils musculaires inertiels permettent une définition plus précise des charges d'entraînement. Science \& Sports, vol. 24, n 2, p. 91-96.

Jidovtseff B., Crielaard J.-M., Cauchy S., Croisier J.-L. (2008). Validité et reproductibilité d'un dynamomètre inertiel basé sur l'accélérométrie. Science \& sports, vol. 23, n² 2, p. 94-97.

Jidovtseff B., Croisier J.-L., Demoulin C., Crielaard J.-M. (2008b). Évaluation inertielle: état de la question et perspectives. Science \& Sports, vol. 23, n 3, p. 107-117.

Jidovtseff B., Croisier J. L., Lhermerout C., Serre L., Sac D., Crielaard J. M. (2006). The concept of iso-inertial assessment: Reproducibility analysis and descriptive data. Isokinetics and Exercise Science, vol. 14, n ${ }^{\circ}$, p. 53-62.

Kavanagh J. J., Menz H. B. (2008). Accelerometry: A technique for quantifying movement patterns during walking. Gait \& Posture, vol. 28, $\mathrm{n}^{\circ}$ 1, p. 1-15.

Koshida S., Urabe Y., Miyashita K., Iwai K., Kagimori A. (2008). Muscular outputs during dynamic bench press under stable versus unstable conditions. The Journal of Strength \& Conditioning Research, vol. 22, $\mathrm{n}^{\circ}$ 5, p. 1584-1588.

Liossis L. D., Forsyth J., Liossis C., Tsolakis C. (2013). the acute effect of upper-body complex training on power output of martial art athletes as measured by the bench press throw exercise. Journal of Human Kinetics, 39, p. 167-175.

Luinge H. J. (2002). Inertial sensing of human movement: Twente University Press.

Luinge H. J., Veltink, P. H. (2004). Inclination measurement of human movement using a 3-D accelerometer with autocalibration. Neural Systems and Rehabilitation Engineering, IEEE Transactions on, vol. 12, $n^{\circ} 1$, p. 112-121.

Mayagoitia R. E., Lötters J. C., Veltink P. H., Hermens H. (2002a). Standing balance evaluation using a triaxial accelerometer. Gait \& Posture, vol. 16, n 1 , p. 55-59.

Mayagoitia R. E., Nene A. V., Veltink P. H. (2002b). Accelerometer and rate gyroscope measurement of kinematics: an inexpensive alternative to optical motion analysis systems. Journal of Biomechanics, vol. 35, $n^{\circ}$ 4, p. 537-542.

Mayhew J. L., Johnson B. D., Lamonte M. J., Lauber D., Kemmler W. (2008). Accuracy of prediction equations for determining one repetition maximum bench press in women before and after resistance training. J Strength Cond Res, vol. 22, n 5, p. 1570-1577.

McMaster D. T., Gill N. D., Cronin J. B., MCGuigan M. R. (2013). Is wireless accelerometry a viable measurement system for assessing vertical jump performance? Sports Technology, vol. 6, n 2, p. 86-96.

Miller C. (1997). Développement des capacités musculaires. Entraînement de la force spécificité et planification, C. M. Thépaut-Mathieu C., Quièvre J. (Ed.), p. 47-84, INSEP.

Moe-NilssenR., Helbostad J. L. (2002). Trunk accelerometry as a measure of balance control during quiet standing. Gait \& Posture, vol. 16, $\mathrm{n}^{\circ} 1$, p. 60-68. 
Morio C., Chavet P., Androuet P., Foissac M., Berton E., Nicol C. (2011). Time course of neuro-mechanical changes underlying stretch-shortening cycle during intermittent exhaustive rebound exercise. European Journal of Applied Physiology, vol. 111, $\mathrm{n}^{\circ}$, p. 2295-2305.

Pereira M. I., Gomes P. S. (2003). Muscular strength and endurance tests: reliability and prediction of one repetition maximum - Review and new evidences. Revista Brasileira de Medicina do Esporte, vol. 9, n 5, p. 336-346.

Rahmani A., Dalleau G., Viale F., Hautier C. A., R L. J. (2000). Validity and reliability of a kinematic device for mesauring the force developed during squatting. Journal of Applied Biomechanics, 16, p. 26-35.

Rontu J.-P., Hannula M. I., Leskinen S., Linnamo V., Salmi J. A. (2010). One-repetition maximum bench press performance estimated with a new accelerometer method. The Journal of Strength \& Conditioning Research, vol. 24, n 8, p. 2018-2025.

Ruben R. M., Molinari M. A., Bibbee C. A., Childress M. A., Harman M. S., Reed K. P., Haff G. G. (2010). The acute effects of an ascending squat protocol on performance during horizontal plyometric jumps. The Journal of Strength \& Conditioning Research, vol. 24, $\mathrm{n}^{\circ}$ 2, p. 358-369.

Sato K., Smith S. L., Sands W. A. (2009). Validation of an accelerometer for measuring sport performance. The Journal of Strength \& Conditioning Research, vol. 23, n 1, p. 341-347.

Sleivert G., Taingahue M. (2004). The relationship between maximal jump-squat power and sprint acceleration in athletes. European Journal of Applied Physiology, vol. 91, $\mathrm{n}^{\circ} 1$, p. 46-52. doi: 10.1007/s00421-003-0941-0

Thompson C. J., Bemben M. G. (1999). Reliability and comparability of the accelerometer as a measure of muscular power. Medicine and science in sports and exercise, vol. $31, \mathrm{n}^{\circ} 6$, p. 897-902.

Veltink P. H., Bussmann H. J., De Vries W., Martens W. L., Van Lummel R. C. (1996). Detection of static and dynamic activities using uniaxial accelerometers. Rehabilitation Engineering, IEEE Transactions on, vol. 4, n 4, p. 375-385.

Vingren J. L., Buddhadev H. H., Hill D. W. (2011). Smith machine counterbalance system affects measures of maximal bench press throw performance. The Journal of Strength \& Conditioning Research, vol. 25, n 7, p. 1951-1956.

Ward D. S., Evenson K. R., Vaughn A., Rodgers A. B., Troiano R. P. (2005). Accelerometer use in physical activity: best practices and research recommendations. Medicine and science in sports and exercise, vol. 37, 11 Suppl, p. S582-588. 